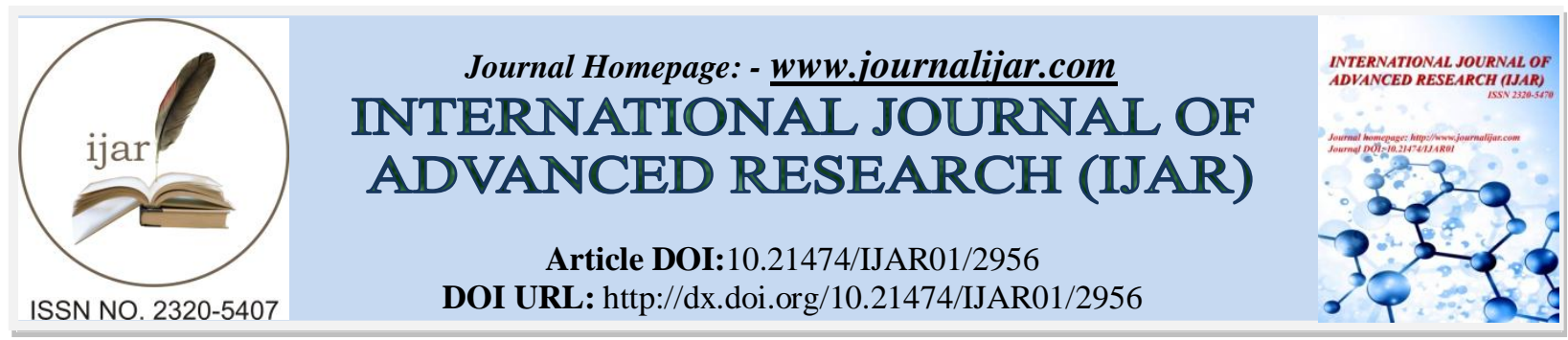

RESEARCH ARTICLE

\title{
ROLE OF S100P AS A NEW PROGNOSTIC MARKER IN WOMEN WITH METASTATIC BREAST CANCER AND ITS CORRELATION WITH BCL2 AND BAX EXPRESSION.
}

\author{
"Lobna A. Abdelaziz" , Hoda F. Ebian ${ }^{2}$,Shereen El Shorbagy ${ }^{3}$, Rham Z.Ahmed ${ }^{3}$, Ola A. Harb ${ }^{4}$, Mariem A. \\ Elfeky $^{4}$, Mohammed E.Eraki ${ }^{5}$ and Loay M. Gertallah ${ }^{5}$. \\ 1. Clinical Oncology and Nuclear Medicine Department, Faculty of Medicine, Zagazig University, \\ 2. Clinical Pathology Department, Faculty of Medicine, Zagazig University. \\ 3. Medical Oncology Department, Faculty of Medicine, Zagazig University. \\ 4. Pathology Department, Faculty of Medicine, Zagazig University. \\ 5. General surgery Department, Faculty of Medicine, Zagazig University.
}

\section{Manuscript Info}

Manuscript History

Received: 29 November 2016

Final Accepted: 26 December 2016

Published: January 2017

Key words:-

Breast cancer, S100P, Bcl2, Bax, apoptosis, prognosis

\section{Abstract}

Background; Breast cancer is the commonest malignancy and the second cause of cancer-related death in women worldwide. Understanding the underlying molecular biology of breast cancer allow better management to decrease its mortality. The S100 P is a member of S100 family of $\mathrm{Ca} 2+$-binding proteins. Apoptosis is a physiologic mechanism of cell death that has been shown to play important role in cancer development. Bcl-2 is an anti-apoptotic gene that has the ability to block apoptotic signals, while Bax is another member of the Bcl-2 family that has an apoptosis-stimulating function.

The aims of our study were to elucidate the prognostic role of S100P, bcl2 and bax in breast cancer, clarify the relation between their expressions and the prognosis of that type of cancer.

Method: The plasma S100P levels ( by ELISA) and expressions of bcl2\& bax ( by Immunohistochemistry) were evaluated in 90 women; 70 metastatic breast cancer patients (MBC), 12 primary breast cancer patients (PBC) and 8 healthy controls, thenwe assessed the prognostic value of S100P, bax, bcl2 in breast cancer patients.

RESULTS: the plasma S100P level was nearly the same for PBC patients and controls, but was higher than that of $\mathrm{MBC}$ patients $(p<0.001)$. There is significant correlations between the level of $\mathrm{S} 100 \mathrm{P}$ with capsular invasion $(\mathrm{p}=0.018)$, stage, bcl2 and bax ( $\mathrm{P}$ $<0.001)$. In MBC there is a significant association between elevation of S100P level, number and site of metastasis $(\mathrm{P}<0.001)$.

$\mathrm{Bcl} 2$ expressions in breast cancer patients had negative significant correlations with grade $(\mathrm{P}<0.001)$, ki67 $(\mathrm{P}<0.021)$, molecular subtype $(\mathrm{P}<0.050)$, stage $(\mathrm{P}<0.001)$, and $\mathrm{S} 100 \mathrm{p}(\mathrm{P}<0.05)$, also its expression in MBC patients was statistically significant with number of metastasis ( $\mathrm{P}=0.014)$.

Bax high expression is statistically significant with grade $(\mathrm{P}=0.003)$, stage $(\mathrm{P}<0.001)$, ER $(\mathrm{P}<0.010)$, PR $(\mathrm{P}<0.023)$, Her-2 neu $(\mathrm{P}<0.002)$,

Corresponding Author:-Lobna A. Abdelaziz.

Address:-Clinical Oncology and Nuclear Medicine Department, Faculty of Medicine, Zagazig 
but in MBC patients it is statistically significant with the site of metastasis $(\mathrm{P}=0.003)$.

The plasma of S100P level is significantly correlated with response to therapy, disease progression $(\mathrm{P}<0.001)$ and Overall survival $(\mathrm{OS})$ of patients $(\mathrm{P}=0.014)$. $\mathrm{Bcl} 2$ expression is significantly correlated with response to therapy $(\mathrm{P}<0.001)$, disease progression $(\mathrm{P} 0.004)$ and $\mathrm{OS}$ $(\mathrm{P}=0.014)$.

Conclusion: S100P, BCL2 and bax are promising prognostic markers in breast cancer patients

Copy Right, IJAR, 2016,. All rights reserved.

\section{Introduction:-}

Breast cancer is the most common neoplasm and the second leading cause of cancer-related death in women worldwide (1). In Egypt, breast cancer represent 33.8\% of all cancer cases in females, and the estimated number of breast cancer in 2015 is 19105 cases (2). Understanding the underlying molecular biology of breast cancer allow earlier diagnosis and efficient treatment options that will help to decrease its mortality (2).

The S100 P is a member of S100 family which is EF-hand superfamily of Ca2+-binding proteins that have intracellular and extracellular functions. It contributes to cancer progression by promoting cell proliferation, survival, angiogenesis, and metastasis. It is over expressed in many types of cancer such as pancreatic cancer, hepatocellular carcinoma, oral squamous cell carcinoma , esophageal cancer,gastric , colorectal cancer, lung cancer , prostatic cancer , ovarian cancer ,cervical cancer . It correlates with poor prognosis in these cancer patients and also could be a promising therapeutic candidate. In addition, many studies suggested an important role of S100P in occurrence of chemoresistance. However, its diagnostic and prognostic value for metastatic breast cancer (MBC) is still unknown (3-6).

Apoptosis is a physiologic mechanism of cell death that has been shown to play a role in the onset and/or development of cancer (7). Defects in the cellular program that control apoptosis can lead to disturbances in tissue homeostasis, i.e., balance of cell proliferation and cell death $(\mathbf{8}, \mathbf{9})$. Most anticancer agents, independently of their mechanisms of action, kill cancer cells by inducing apoptosis in response to a drug-induced damage. Alterations in the regulatory mechanisms of apoptosis are responsible not only for the progression of cancer, but also for different response to treatment (10). Apoptosis is controlled by several genes. Among the most crucial regulators of this process are members of the Bcl-2 gene family. Bcl-2 is an anti-apoptotic gene that was first identified in follicular non- Hodgkin lymphoma (11). Bax is another member of the Bcl-2 family, but in contrast to Bcl-2 it has an apoptosis-stimulating function (12).

\section{The aims of our study:-}

were to elucidate the role of plasma S100P level in breast cancer prognosis, evaluate the immunohistochemical expression of bcl 2 \& baxin breast cancer patients, clarify the relation between their expressions and patient prognosis, and also to correlate plasma S100P level with bcl2 and bax immunohistochemical expressions in our patients.

\section{Patients \& method:-}

In our study, 90 women were included; 70 metastatic breast cancer patients (MBC), 12 primary breast cancer patients (PBC), and 8 healthy controls, the study was conducted in Clinical Oncology and Nuclear Medicine, Medical Oncology, Clinical Pathology, Pathology, General Surgery departments, faculty of medicine, Zagazig University, Egypt. Informed consent was obtained from all cases; the study protocol was approved by the Ethical Committee of Faculty of Medicine, Zagazig University. The patients' specimens and data were collected from March 2011 to February 2013 and were followed up till March 2016.

\section{Plasma S100P measurement:-}

Plasma S100P level was measured using S100P ELISA kit (ElabScince, prouduct code E-E1-H1294) and an ELISA plate reader b (Tecan-Austria GM bit.8 Gro dig. Austria, following the manuel of the procedure. All plasma samples were measured in duplicates. Correlation coefficient between duplicate: 0.99 , detection range $(0.313-20 \mathrm{ng} / \mathrm{ml})$, sensitivity: 0.188 .

Sample collection and storage collect plasma using EDTA as antianticoagulant, centrifugation the sample at 1300g at 2-8C for 20mints of collection the plasma. Collect the supernatant and stored at -80 , then before the assay the sample kept at RT (8-25C).Determine optical density (OD) value of each well at microplate reader (450nm). 
Tissue specimens: Formalin fixed paraffin embedded blocks of PBC and MBC patients were collected. The seventh edition of the American Joint Committee on Cancer staging system (AJCC-7) classification was used for pathologic staging (13) and the Nottingham (Elston-Ellis) modification of the Scarff -Bloom-Richardson grading system was used for pathologic grading (14).

Immunohistochemical staining: Immunohistochemical staining was carried out using the streptavidin-biotin immunoperoxidase technique (15), the slides were incubated with mouse monoclonal Anti-Bcl-2 antibody [Bcl2/100] ab117115 was used at a dilution of 1:100 and primary rabbit polyclonal Anti-Bax antibody ab10813 diluted 1:1000 (Abcam, Cambridge, MA, USA) at $4^{\circ} \mathrm{C}$ overnight. Sections from normal tonsils were used as positive control for bcl2, sections from normal colon for bax and the negative control is by adding non-immune serum instead of the primary antibodies.

\section{Evaluation of immunohistochemical expressions of Bcl2 proteins:}

Cytoplasmic staining of bcl-2was scored as followed; bcl2 negative-no tumor cells stain or weak heterogeneous positive stain in less than $10 \%$ of tumor cells and bcl-2 positive-more than $10 \%$ of tumor cells stained (16).

\section{Evaluation of immunohistochemical expression of bax:}

Bax cytoplasmic expression was scored as positive if at least $20 \%$ of tumor cells showed clear cytoplasmic immunostaining (17).

\section{Statistical Analysis:-}

Continuous variables were expressed as the mean \pm SD \& median (range), and the categorical variables were expressed as a number(percentage). Continuous variables were checked for normality by using Shapiro-Wilk test. Percent of categorical variables were compared using Pearson's Chi-square test or Fisher's exact test when was appropriate. Receiver operating characteristic (ROC) curve analysis was used to identify optimal cut-off value of S100 level with maximum sensitivity and specificity for discrimination between breast cancer and control. Overall Survival (OS) was calculated as the time from diagnosis to death or the most recent follow-up contact (censored). Progression Free Survival (PFS) was calculated as the time from start of treatment to date of progression or the most recent follow-up contact that patient was known as progression free. Stratification of OS and PFS was done according markers. These time-to-event distributions were estimated using the method of Kaplan-Meier plot, and compared using two-sided exact log-rank test. All tests were two sided. A p-value <0.05 was considered significant. All statistics were performed using SPSS 22.0 for windows (SPSS Inc., Chicago, IL, USA) and MedCalc windows (MedCalc Software bvba 13, Ostend, Belgium).

\section{Results:- \\ Patient Characteristics}

Our study included 70 MBC patients, their age range was (29-65) years, mean + -SD was $47.25+-9.77$. Forty four patients $(62.9 \%)$ were less than 50 years while 26 patients $(37.1 \%)$ were more than 50 years .Premenopausal and post menopausal patients were presented in $30(42.9 \%)$ and $40(57.1 \%)$ respectively. Fifty five patients (78.6\%) had IDC ,12 patients $(17.1 \%)$ had ILC while medullary and mucinous carcinoma were presented in only one and two patients respectively. The majority of our patients (70\%) had G2 whereas only 30\% had G3.Capsular invasion and positive Ki67 were demonstrated in $15(21.4 \%)$ patients and $20(28.6 \%)$ patients respectively. The majority of our patients had Luminal A subtype (57.1\%) but luminal B and Her-2 amplified subtypes were presented in (15.7\%) for each one. whereas the minority of our patients $(15.7 \%)$ had triple negative subtype .T1,T2,T3 and T4 were present in $12,19,12$ and 17 patients respectively where N0, N1,N2 and N3 were present in 16,15,2 and 37 patients respectively.S100P mean were $13.27+-$ 6.66,the median(range) were 13.10 (8.2-19.3).Negative bcl2 and bax expression were present in 47 and 23 patients respectively. while positive bcl2 and bax expression were present in 23 and 47 respectively(Table 2).

\section{Comparison between the studied groups as regard the 3 studied markers}

-The median of plasma S100 P level was nearly the same for PBC patients and healthy control, in addition its level in MBC patients was higher than both PBC patients and healthy control (near the double) (p1<0.001 between MBC vs Non-MBC; p2 $<0.001$ between MBC vs control) both of them are significant while p3 $=0.834$ between Non-MBC vs control (non significant).

-Regarding Bcl2 immunoexpression positive expression was found in 23 (32.9\%) of MBC, 11 (91.7\%) of Non-MBC and in $8(100 \%)$ of the normal breast tissue (p1<0.001 between MBC vs Non-MBC; p2<0.001 between MBC vs control) both of them are significant while p3 $=0.402$ between Non-MBC vs control (non significant). 
-Regarding Bax immunoexpression; positive expression was found in $47(67.1 \%)$ of cases of MBC but no positive expression was detected in either Non-MBC or in the normal breast tissue ( $\mathrm{p} 3<0.001)$. ( $\mathrm{p} 1<0.001$ between MBC vs NonMBC; p2 $<0.001$ between MBC vs control; p3 $<0.001$ between Non-MBC vs control all of them are significant (Table 1).

Table (1): Comparison between the studied groups as regard the 3 studied markers

\begin{tabular}{|c|c|c|c|c|c|c|c|c|c|c|}
\hline \multirow[t]{2}{*}{ Markers } & \multicolumn{2}{|c|}{$\begin{array}{l}\text { MBC } \\
(\mathrm{N}=70)\end{array}$} & \multicolumn{2}{|c|}{$\begin{array}{l}\text { Non-MBC } \\
(\mathrm{N}=12)\end{array}$} & \multicolumn{2}{|c|}{$\begin{array}{l}\text { Control } \\
(\mathrm{N}=100)\end{array}$} & \multirow[t]{2}{*}{ p-value } & \multirow[t]{2}{*}{$\mathrm{p}^{1}$} & \multirow[t]{2}{*}{$p^{2}$} & \multirow[t]{2}{*}{$\mathrm{p}^{3}$} \\
\hline & No. & $(\%)$ & No. & $(\%)$ & No. & $(\%)$ & & & & \\
\hline \multicolumn{11}{|l|}{ S100 } \\
\hline Mean \pm SD & 13.27 & \pm 0.66 & 6.88 & \pm 0.04 & 6.53 & \pm 0.93 & \multirow[t]{2}{*}{$<0.001 *$} & \multirow[t]{2}{*}{ k0.001 } & \multirow[t]{2}{*}{$<0.001$} & \multirow[t]{2}{*}{0.834} \\
\hline Median (Range) & 13.10 & $(8.2-19.3)$ & 6.80 & $(4.9-8.5)$ & 6.75 & $(4.9-7.6)$ & & & & \\
\hline \multicolumn{11}{|l|}{$\mathrm{Bcl} 2$} \\
\hline Negative & 47 & $(67.1 \%)$ & 1 & $(8.3 \%)$ & 0 & $(0 \%)$ & \multirow[t]{2}{*}{ k0.001\$ } & \multirow[t]{2}{*}{$<0.001$} & \multirow[t]{2}{*}{$<0.001$} & \multirow[t]{2}{*}{0.402} \\
\hline Positive & 23 & $(32.9 \%)$ & 11 & $(91.7 \%)$ & 8 & $(100 \%)$ & & & & \\
\hline \multicolumn{11}{|l|}{ Bax } \\
\hline Negative & 23 & $(32.9 \%)$ & 12 & $(100 \%)$ & 8 & $(100 \%)$ & \multirow[t]{2}{*}{ k0.001t } & \multirow[t]{2}{*}{$<0.001$} & \multirow[t]{2}{*}{$<0.001$} & \multirow[t]{2}{*}{--} \\
\hline Positive & 47 & $(67.1 \%)$ & 0 & $(0 \%)$ & 0 & $(0 \%)$ & & & & \\
\hline
\end{tabular}

Table (2): Effect of clinopathological parameters onimmunohistochemical staining for( Bcl2, bax )and plasma S100p level in metastatic breast cancer patients $(\mathrm{N}=70)$.

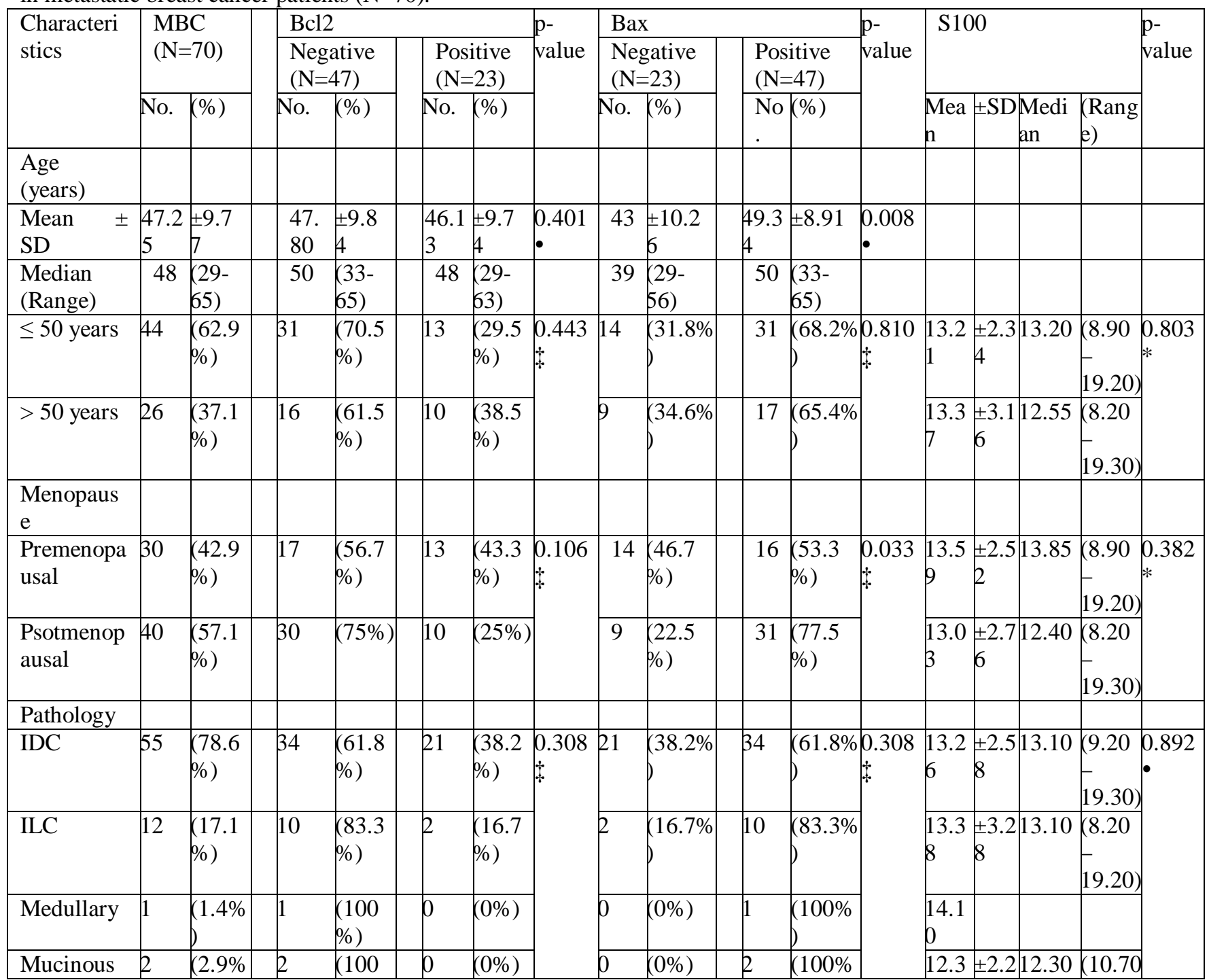




\begin{tabular}{|c|c|c|c|c|c|c|c|c|c|c|c|c|c|c|c|c|c|}
\hline & & ) & & $\%)$ & & & & & & & ) & & & 6 & & (13.90) & \\
\hline Grade & & & & & & & & & & & & & & & & & \\
\hline Grade II & 49 & $(70 \%)$ & 27 & $\begin{array}{l}55.1 \\
\%)\end{array}$ & 22 & $\begin{array}{l}(44.9 \\
\%)\end{array}$ & $\begin{array}{l}0.001 \\
+\end{array}$ & 22 & (44.9\% & 27 & $f^{55.1 \%}$ & $\begin{array}{l}0.001 \\
+ \\
+\end{array}$ & 13.2 & \begin{tabular}{|l|}
2.8 \\
3
\end{tabular} & 12.90 & \begin{tabular}{|l}
$(8.90$ \\
$19.30)$
\end{tabular} & 0.827 \\
\hline Grade III & 21 & $(30 \%)$ & 20 & $\begin{array}{l}(95.2 \\
\%)\end{array}$ & 1 & (4.8\% & & 1 & $(4.8 \%)$ & 20 & $95.2 \%$ & & $5^{13.2}$ & \pm 2.2 & 13.30 & \begin{tabular}{|l}
$(8.20$ \\
$17.90)$ \\
\end{tabular} & \\
\hline $\begin{array}{l}\text { Capsular } \\
\text { invasion }\end{array}$ & & & & & & & & & & & & & & & & & \\
\hline Absent & 55 & $\begin{array}{l}(78.6 \\
\%)\end{array}$ & 34 & $\begin{array}{l}(61.8 \\
\%)\end{array}$ & 21 & $\left(\begin{array}{l}38.2 \\
\%)\end{array}\right.$ & $\begin{array}{l}0.119 \\
+ \\
+\end{array}$ & 21 & $(38.2 \%$ & 35 & )$^{61.8 \%}$ & $\begin{array}{l}0.119 \\
t \\
+\end{array}$ & $\begin{array}{l}12.7 \\
6\end{array}$ & $\begin{array}{l} \pm 2.4 \\
5\end{array}$ & 12.80 & $\left(\begin{array}{l}(8.20 \\
-19.20)\end{array}\right.$ & $\begin{array}{l}0.002 \\
*\end{array}$ \\
\hline Present & 15 & $\begin{array}{l}(21.4 \\
\%)\end{array}$ & 13 & $\begin{array}{l}86.7 \\
\%)\end{array}$ & 2 & $\begin{array}{l}(13.3 \\
\%)\end{array}$ & & 2 & (13.3\% & 13 & $86.7 \%$ & & $2^{15.1}$ & $\begin{array}{l} \pm 2.6 \\
2\end{array}$ & 14.90 & \begin{tabular}{|l}
$(10.90$ \\
$19.30)$ \\
\end{tabular} & \\
\hline KI-67 & & & & & & & & & & & & & & & & & \\
\hline Negative & & $(71.4$ & 30 & $(60 \%)$ & 20 & $(40 \%)$ & $\begin{array}{l}0.044 \\
\vdots \\
\vdots\end{array}$ & 22 & $(44 \%)$ & 28 & $(56 \%)$ & $\begin{array}{l}0.002 \\
+ \\
+\end{array}$ & 13.1 & \begin{tabular}{|l}
2.7 \\
9
\end{tabular} & 12.90 & $\begin{array}{l}(8.90 \\
19.30) \\
\end{array}$ & 0.424 \\
\hline Positive & 20 & $(28.6$ & 17 & $(85 \%)$ & 3 & $(15 \%)$ & & 1 & $(5 \%)$ & 19 & $(95 \%)$ & & $\begin{array}{l}13.5 \\
1\end{array}$ & \begin{tabular}{|l|} 
\pm 2.3 \\
2
\end{tabular} & 13.75 & $\begin{array}{l}(8.20 \\
-18.40) \\
\end{array}$ & \\
\hline $\begin{array}{l}\text { Molecular } \\
\text { type }\end{array}$ & & & & & & & & & & & & & & & & & \\
\hline Luminal A & 40 & $\begin{array}{l}(57.1 \\
\%)\end{array}$ & 22 & $(55 \%)$ & 18 & $(45 \%)$ & $\begin{array}{l}0.050 \\
+\end{array}$ & 22 & $(55 \%)$ & 18 & $(45 \%)$ & $\begin{array}{l}<0.00 \\
1 \dagger\end{array}$ & $2^{13.0}$ & \begin{tabular}{|l|}
2.7 \\
6
\end{tabular} & 12.40 & $\begin{array}{l}(8.90 \\
19.30)\end{array}$ & $\begin{array}{l}0.326 \\
\bullet\end{array}$ \\
\hline Luminal B & 11 & $\begin{array}{l}(15.7 \\
\%)\end{array}$ & 8 & $\begin{array}{l}(72.7 \\
\%)\end{array}$ & $\beta$ & $\begin{array}{l}(27.3 \\
\%)\end{array}$ & & 1 & $(9.1 \%)$ & 10 & $(90.9 \%$ & & $\int^{14.3}$ & \begin{tabular}{|l|} 
\pm 3.1 \\
5
\end{tabular} & 14.30 & \begin{tabular}{|l}
$(8.20$ \\
$18.60)$ \\
\end{tabular} & \\
\hline Triple -ve & 8 & $\begin{array}{l}(11.4 \\
\%)\end{array}$ & 8 & $\begin{array}{l}(100 \\
\%)\end{array}$ & 0 & $(0 \%)$ & & 0 & $(0 \%)$ & 8 & $(100 \%$ & & $\begin{array}{l}13.6 \\
5\end{array}$ & \begin{tabular}{|l|}
3 \\
3
\end{tabular} & 13.30 & $\begin{array}{l}(12.10 \\
15.30) \\
\end{array}$ & \\
\hline $\begin{array}{l}\text { HER2 } \\
\text { amplified }\end{array}$ & 11 & $\begin{array}{l}(15.7 \\
\%)\end{array}$ & 9 & $\begin{array}{l}(81.8 \\
\%)\end{array}$ & 2 & $\begin{array}{l}(18.2 \\
\%)\end{array}$ & & 0 & $(0 \%)$ & 11 & $(100 \%$ & & $8^{12.8}$ & \begin{tabular}{|l|}
2.5 \\
4
\end{tabular} & 11.70 & $\begin{array}{l}(9.50 \\
18.40) \\
\end{array}$ & \\
\hline $\mathrm{T}$ & & & & & & & & & & & & & & & & & \\
\hline T0 & 10 & $\begin{array}{l}(14.3 \\
\%)\end{array}$ & $\beta$ & $(30 \%)$ & 7 & $(70 \%)$ & $\begin{array}{l}<0.00 \\
1 \S\end{array}$ & 7 & $(70 \%)$ & 3 & $(30 \%)$ & $\begin{array}{l}0.00 \\
1 \S\end{array}$ & & \begin{tabular}{|l|}
1.8 \\
7
\end{tabular} & 13.15 & $\begin{array}{l}(10.20 \\
16.20) \\
\end{array}$ & * \\
\hline $\mathrm{T} 1$ & 12 & $\begin{array}{l}(17.1 \\
\%)\end{array}$ & 8 & $\begin{array}{l}(66.7 \\
\%)\end{array}$ & 4 & $\begin{array}{l}(33.3 \\
\%)\end{array}$ & & 4 & (33.3\% & 8 & $66.7 \%$ & & $\begin{array}{l}13.4 \\
5\end{array}$ & $\begin{array}{l} \pm 3.5 \\
4\end{array}$ & 12.40 & $\begin{array}{l}(8.90 \\
19.20) \\
\end{array}$ & \\
\hline $\mathrm{T} 2$ & 19 & $\begin{array}{l}(27.1 \\
\%)\end{array}$ & 8 & $\begin{array}{l}(42.1 \\
\%)\end{array}$ & 11 & $\begin{array}{l}(57.9 \\
\%)\end{array}$ & & 10 & $(52.6 \%$ & 9 & (47.4\% & & $0^{12.6}$ & \begin{tabular}{|l|} 
\pm 2.2 \\
5
\end{tabular} & 12.20 & $\begin{array}{l}(9.70 \\
17.90) \\
\end{array}$ & \\
\hline T3 & 12 & $(17.1$ & 11 & $\begin{array}{l}(91.7 \\
\%)\end{array}$ & 1 & $8.3 \%$ & & 1 & $(8.3 \%)$ & 11 & $91.7 \%$ & & $\begin{array}{l}12.3 \\
8\end{array}$ & $\begin{array}{l} \pm 1.9 \\
5\end{array}$ & 12.70 & $\begin{array}{l}(8.20 \\
14.70) \\
\end{array}$ & \\
\hline $\mathrm{T} 4$ & 17 & $(24.3$ & 17 & $\begin{array}{l}(100 \\
\%)\end{array}$ & 0 & $(0 \%)$ & & 1 & $(5.9 \%)$ & 16 & $9^{94.1 \%}$ & & $4^{14.7}$ & $\begin{array}{l} \pm 2.8 \\
2\end{array}$ & 14.90 & \begin{tabular}{|l|}
$(9.50$ \\
$19.30)$ \\
\end{tabular} & \\
\hline $\mathrm{N}$ & & & & & & & & & & & & & & & & & \\
\hline
\end{tabular}




\begin{tabular}{|c|c|c|c|c|c|c|c|c|c|c|c|c|c|c|c|c|c|}
\hline N0 & 16 & $\begin{array}{l}(22.9 \\
\%)\end{array}$ & 1 & $6.3 \%$ & 15 & $\begin{array}{l}93.8 \\
\%)\end{array}$ & $\begin{array}{l}<0.00 \\
1 \S\end{array}$ & 14 & $87.5 \%$ & 2 & $(12.5 \%$ & $\begin{array}{l}<0.00 \\
1 \S\end{array}$ & & \pm 2.0 & 12.25 & \begin{tabular}{|l|}
9.70 \\
$15.60)$ \\
\end{tabular} & 0.261 \\
\hline N1 & 15 & $\begin{array}{l}(21.4 \\
\%)\end{array}$ & 7 & $\begin{array}{l}(46.7 \\
\%)\end{array}$ & 8 & $\begin{array}{l}(53.3 \\
\%)\end{array}$ & & 9 & $(60 \%)$ & 6 & $(40 \%)$ & & $8^{12.9}$ & $\begin{array}{l} \pm 3.0 \\
9\end{array}$ & 12.10 & \begin{tabular}{|l|}
$(8.90$ \\
$19.20)$ \\
\end{tabular} & \\
\hline $\mathrm{N} 2$ & 2 & $(2.9 \%$ & 2 & $\begin{array}{l}(100 \\
\%)\end{array}$ & 0 & $(0 \%)$ & & 0 & $(0 \%)$ & 2 & $(100 \%$ & & 16 & $\begin{array}{l} \pm 3.3 \\
9\end{array}$ & 16 & $\begin{array}{l}(13.60 \\
18.40) \\
\end{array}$ & \\
\hline N3 & 37 & $\begin{array}{l}(52.9 \\
\%)\end{array}$ & 37 & $\begin{array}{l}(100 \\
\%)\end{array}$ & 0 & $(0 \%)$ & & 0 & $(0 \%)$ & 37 & $(100 \%$ & & & \pm 2.6 & 13.40 & \begin{tabular}{|l|}
$(8.20$ \\
$19.30)$ \\
\end{tabular} & \\
\hline $\mathrm{Bcl}$ & & & & & & & & & & & & & & & & & \\
\hline Negative & 47 & $\begin{array}{l}(67.1 \\
\%)\end{array}$ & & & & & & 8 & (17.1\% & 39 & $82.9 \%$ & $\begin{array}{l}<0.00 \\
1 \dagger\end{array}$ & $14.1=$ & $\begin{array}{l} \pm 2.2 \\
8\end{array}$ & 13.90 & \begin{tabular}{|l}
$(10.70$ \\
$-19.30)$ \\
\end{tabular} & $\begin{array}{l}0<0.00 \\
1 \bullet\end{array}$ \\
\hline Positive & 23 & $\begin{array}{l}(32.9 \\
\%)\end{array}$ & & & & & & 15 & (65.2\% & 8 & $(34.8 \%$ & & & $\begin{array}{l} \pm 2.5 \\
0\end{array}$ & 10.80 & \begin{tabular}{|l|}
$(8.20$ \\
$19.20)$ \\
\end{tabular} & \\
\hline Bax & & & & & & & & & & & & & & & & & \\
\hline Negative & 23 & $\begin{array}{l}(32.9 \\
\%)\end{array}$ & 8 & $\begin{array}{l}(34.8 \\
\%)\end{array}$ & 15 & $\begin{array}{l}(65.2 \\
\%)\end{array}$ & $\begin{array}{l}<0.00 \\
1 \ddagger\end{array}$ & & & & & & 12.9 & & 12.30 & \begin{tabular}{|l|}
$(8.90$ \\
$19.20)$ \\
\end{tabular} & $\begin{array}{l}0.450 \\
*\end{array}$ \\
\hline Positive & 47 & $\begin{array}{l}(67.1 \\
\%)\end{array}$ & 39 & $(83 \%)$ & 8 & $(17 \%)$ & & & & & & & $\begin{array}{l}13.4 \\
4\end{array}$ & \pm 2.7 & 13.30 & \begin{tabular}{|l|}
$(8.20$ \\
$19.30)$ \\
\end{tabular} & \\
\hline S100 & & & & & & & & & & & & & & & & & \\
\hline $\begin{array}{ll}\text { Mean } & \pm \\
\text { SD } & \end{array}$ & $\begin{array}{l}13.2 \\
7\end{array}$ & \pm 0.66 & $\begin{array}{l}14.1 \\
4\end{array}$ & \pm 2.28 & $\begin{array}{l}11.4 \\
8\end{array}$ & \pm 2.50 & $\begin{array}{l}<0.00 \\
1 \bullet\end{array}$ & $2^{12.9}$ & \pm 2.52 & $\begin{array}{l}13 . \\
4\end{array}$ & \pm 2.73 & $\begin{array}{l}0.450 \\
*\end{array}$ & & & & & \\
\hline $\begin{array}{l}\text { Median } \\
\text { (Range) }\end{array}$ & $\begin{array}{l}13.1 \\
0\end{array}$ & $\begin{array}{l}(8.2- \\
19.3)\end{array}$ & $\begin{array}{l}13.9 \\
0\end{array}$ & \begin{tabular}{|l}
$(10.70$ \\
$19.30)$ \\
\end{tabular} & $0^{10.8}$ & \begin{tabular}{|l|}
$(8.20$ \\
$19.20)$ \\
\end{tabular} & & $\begin{array}{l}12.3 \\
0\end{array}$ & $\begin{array}{l}(8.90 \\
19.20)\end{array}$ & $\begin{array}{l}13 . \\
0\end{array}$ & $\begin{array}{l}(8.20 \\
19.30)\end{array}$ & & & & & & \\
\hline
\end{tabular}

Continuous variables were expressed as mean \pm SD \& median (range); categorical variables were expressed as number(percentage); *Independent samples Student's test; • Mann Whitney U test; $\$$ Chi-square test; $§$ Chi-square test for trend; $\mathrm{p}<0.05$ is significant.

\section{Effect of clinicopathological parameters on plasma S100p level in breast cancer patients}

plasma level of S100 in all breast cancer patients showed statistical correlation with capsular invasion ( $\mathrm{p}=0.018), \mathrm{T}$ ( $\mathrm{p}=0.031)$, stage, bcl2 and bax expression $(\mathrm{P}<0.001$ for each of them ), while $\mathrm{S} 100$ level in metastatic breast cancer patients showed significant correlation with capsular invasion and $b c 12(\mathrm{P}=0.002 \&<0.001$ respectively). Furthermore, there is a significant association between elevation of S100P level and both the number and site of metastasis $(\mathrm{P}<0.001$ for both); $\{$ presence of liver metastasis $\mathrm{P}=0.002$, brain metastasis $\mathrm{P}=0.009$ and bone metastasis $\mathrm{P}<0.001\}$ (Table 2, 3 ).

\section{Correlation between clinicopathological parameters and Bcl2 expression in breast cancer patients}

Bcl2 was cytoplasmic and its protein expressions in breast cancer tissues were lower than those in the relatively healthy and adjacent breast tissues .Bcl2 was positively expressed in $41.5 \%$ of all studied breast cancer patients, 32.9\% of MBC, and $91.7 \%$ of $\mathrm{PBC}$. Moreover, its low expression in all breast cancer patients was significantly negatively correlated with grade ( $\mathrm{P} 0.001)$, ki67 ( $\mathrm{P} 0.021)$, molecular subtype ( $\mathrm{P} 0.050)$, and each one of $\mathrm{T}, \mathrm{N}$, stage , BAX ( $\mathrm{P}<0.001$ for each of them ), S100p ( $\mathrm{P}<0.05)$. But its expression in MBC patients was statistically significant with, ki67 (P 0.044), molecular subtype ( $\mathrm{P} 0.050)$, and each one of grade, $\mathrm{T}, \mathrm{N}, \mathrm{BAX}(\mathrm{P}<0.001$ for each of them), number of metastasis $(\mathrm{P}=0.014)$ and presence of bone metastasis $(\mathrm{P}=0.013)($ Table 2,3 $)$. 


\section{Correlation between clinicopathological parameters and bax expression}

Bax was cytoplasmic and its protein expression in breast cancer tissues was significantly higher than that in the relatively healthy, adjacent breast tissues. . Bax was positively expressed in $57.3 \%$ of all studied breast cancer patients, $67 \%$ of MBC, while all $\mathrm{PBC}$ didn't express bax.. Furthermore, its high expression is statistically significant with grade $(\mathrm{P}=0.003)$,every one of molecular subtype , $\mathrm{T}, \mathrm{N}$ and stage $(\mathrm{P}<0.001$ for each $)$, ki67 $(\mathrm{P}<0.004), \mathrm{ER}(\mathrm{P}<0.010), \mathrm{PR}(\mathrm{P}<0.023)$,Her-2 neu $(\mathrm{P}<0.002)$, both bcl2 and BAX ( $<<0.001$ for both) in all breast cancer patients . However in MBC patients it is statistically significant with grade $(\mathrm{P}=0.001)$, every one of molecular subtype, $\mathrm{T}, \mathrm{N}(\mathrm{P}<0.001$ for each $)$, ki67 $(\mathrm{P}<0.002)$, site of metastasis $(\mathrm{P}=0.003)$, presence of brain metastasis $(\mathrm{P}=0.004)$ and lung metastasis $(\mathrm{P}=0.032)\}$ (Table 2,3).

Table (3): correlations between distant metastasis and immunohistochemical expressions( bcl2, bax) and plasma S100 level in metastatic breast cancer patients

\begin{tabular}{|c|c|c|c|c|c|c|c|c|c|c|c|c|c|c|c|c|c|}
\hline \multirow{3}{*}{$\begin{array}{l}\text { Characteristi } \\
\text { cs }\end{array}$} & \multirow{2}{*}{\multicolumn{2}{|c|}{$\begin{array}{l}\mathrm{MBC} \\
(\mathrm{N}=70)\end{array}$}} & \multicolumn{4}{|c|}{$\mathrm{Bcl} 2$} & \multirow{3}{*}{ p-value } & \multicolumn{4}{|c|}{ Bax } & \multirow[t]{3}{*}{$p$-value } & \multirow{2}{*}{\multicolumn{4}{|c|}{ S100 }} & \multirow{3}{*}{$\begin{array}{l}\mathrm{p}- \\
\text { value }\end{array}$} \\
\hline & & & \multirow{2}{*}{\multicolumn{2}{|c|}{\begin{tabular}{|l|}
$\begin{array}{l}\text { Negati } \\
\text { ve } \\
(\mathrm{N}=47)\end{array}$ \\
No $(\%)$
\end{tabular}}} & \multirow{2}{*}{\multicolumn{2}{|c|}{\begin{tabular}{|l|}
$\begin{array}{l}\text { Positiv } \\
\text { e } \\
(\mathrm{N}=23)\end{array}$ \\
No $(\%)$
\end{tabular}}} & & \multicolumn{2}{|c|}{$\begin{array}{l}\text { Negativ } \\
\mathrm{e} \\
(\mathrm{N}=23)\end{array}$} & \multicolumn{2}{|c|}{$\begin{array}{l}\text { Positive } \\
(\mathrm{N}=47)\end{array}$} & & & & & & \\
\hline & \multicolumn{2}{|c|}{ No $(\%)$} & & & & & & No & $(\%)$ & & $(\%)$ & & Mea & $\pm \mathrm{SD}$ & $\begin{array}{l}\text { Medi } \\
\text { an }\end{array}$ & $\begin{array}{l}\text { (Rang } \\
\text { e) }\end{array}$ & \\
\hline $\begin{array}{l}\text { Time of } \\
\text { metastasis }\end{array}$ & & & & & & & & & & & & & & & & & \\
\hline Synchronus & & $\begin{array}{l}(17.1 \\
\%)\end{array}$ & 11 & $\begin{array}{l}(91.7 \\
\%)\end{array}$ & 1 & $8.3 \%$ & $\begin{array}{l}0.08 \\
8 \$\end{array}$ & 1 & $8.3 \%$ & 11 & $\begin{array}{l}(91.7 \\
\%)\end{array}$ & $\begin{array}{l}0.08 \\
8 \ddagger\end{array}$ & $\begin{array}{l}13.8 \\
0\end{array}$ & $\begin{array}{l}+1.8 \\
1\end{array}$ & 13.70 & \begin{tabular}{|l|}
$(11.50$ \\
$17.80)$
\end{tabular} & $\begin{array}{l}0.448 \\
* \\
\end{array}$ \\
\hline Metachronus & 58 & $\begin{array}{l}(82.9 \\
\%)\end{array}$ & 36 & $\begin{array}{l}(62.1 \\
\%)\end{array}$ & 22 & $\begin{array}{l}(37.9 \\
\%)\end{array}$ & & & $\begin{array}{l}37.9 \\
\%)\end{array}$ & 36 & $\begin{array}{l}(62.1 \\
\%)\end{array}$ & & $\begin{array}{l}13.1 \\
6\end{array}$ & $\begin{array}{l}+2.8 \\
0\end{array}$ & 12.90 & \begin{tabular}{|l|}
$(8.20$ \\
$-19.30)$
\end{tabular} & \\
\hline $\begin{array}{ll}\text { No. } & \text { o } \\
\text { metastasis } & \\
\end{array}$ & & & & & & & & & & & & & & & & & \\
\hline Single & 47 & $\begin{array}{l}(67.1 \\
\%)\end{array}$ & 27 & $\begin{array}{l}57.4 \\
\%)\end{array}$ & 20 & $\begin{array}{l}(42.6 \\
\%)\end{array}$ & $\begin{array}{l}0.01 \\
4 \ddagger\end{array}$ & $\begin{array}{l}1 \\
7\end{array}$ & $\begin{array}{l}36.2 \\
\%)\end{array}$ & 30 & $\begin{array}{l}(63.8 \\
\%)\end{array}$ & $\begin{array}{l}0.39 \\
9 \ddagger\end{array}$ & $\begin{array}{l}12.2 \\
3\end{array}$ & $\begin{array}{l}+2.2 \\
1\end{array}$ & 12.10 & \begin{tabular}{|l|}
$(8.20$ \\
$19.20)$ \\
\end{tabular} & $\begin{array}{l}<0.00 \\
1^{*}\end{array}$ \\
\hline Multiple & 23 & $\begin{array}{l}(32.9 \\
\%)\end{array}$ & 20 & $(87 \%)$ & 3 & $(13 \%)$ & & 6 & $\begin{array}{l}(26.1 \\
\%)\end{array}$ & 17 & $\begin{array}{l}(73.9 \\
\%)\end{array}$ & & 2.21 & +2.2 & 15.20 & \begin{tabular}{|l}
$(11.10$ \\
$-19.30)$ \\
\end{tabular} & \\
\hline $\begin{array}{l}\text { Type of } \\
\text { metastasis }\end{array}$ & & & & & & & & & & & & & & & & & \\
\hline Bone & 27 & $\begin{array}{l}38.6 \\
\%)\end{array}$ & 14 & $\begin{array}{l}(51.9 \\
\%)\end{array}$ & 13 & $\begin{array}{l}(48.1 \\
\%)\end{array}$ & $\begin{array}{l}0.08 \\
1 \$\end{array}$ & $\begin{array}{l}1 \\
1\end{array}$ & $\begin{array}{l}(40.7 \\
\%)\end{array}$ & 16 & $\begin{array}{l}(59.3 \\
\%)\end{array}$ & $\begin{array}{l}0.00 \\
3 \dagger\end{array}$ & $b^{11.4}$ & $\begin{array}{l}+1.6 \\
2\end{array}$ & 11.20 & \begin{tabular}{|l|}
$(8.20$ \\
$14.80)$ \\
\end{tabular} & $\begin{array}{l}<0.00 \\
1 \bullet\end{array}$ \\
\hline Brain & 7 & $(10 \%)$ & 4 & $\begin{array}{l}(57.1 \\
\%)\end{array}$ & 3 & $\begin{array}{l}(42.9 \\
\%)\end{array}$ & & 6 & $\begin{array}{l}85.7 \\
\%)\end{array}$ & 1 & $\begin{array}{l}(14.3 \\
\%)\end{array}$ & & $2^{15.7}$ & $\begin{array}{l}+2.5 \\
0\end{array}$ & 15.30 & $\begin{array}{l}(11.80 \\
-19.20)\end{array}$ & \\
\hline Lung & 16 & $\begin{array}{l}(22.9 \\
\%)\end{array}$ & 14 & $\begin{array}{l}87.5 \\
\%)\end{array}$ & 2 & $\begin{array}{l}(12.5 \\
\%)\end{array}$ & & 1 & $6.3 \%$ & 15 & $\begin{array}{l}(93.8 \\
\%)\end{array}$ & & $\left.\right|^{13.4}$ & $\begin{array}{l}+2.0 \\
8\end{array}$ & 13.55 & \begin{tabular}{|l|}
$(9.50$ \\
- \\
$17.50)$
\end{tabular} & \\
\hline Liver & 5 & $(7.1 \%$ & 5 & $(100 \%$ & 0 & $(0 \%)$ & & 0 & $(0 \%)$ & 5 & $\begin{array}{l}(100 \\
\%)\end{array}$ & & $\begin{array}{l}17.5 \\
6\end{array}$ & +2.0 & 17.90 & \begin{tabular}{|l}
$(14.10$ \\
$19.30)$
\end{tabular} & \\
\hline Lung+Liver & 2 & $(2.9 \%$ & 2 & $(100 \%$ & 0 & $(0 \%)$ & & 0 & $(0 \%)$ & 2 & $\begin{array}{l}(100 \\
\%)\end{array}$ & & $0^{16.8}$ & $\begin{array}{l} \pm 2.2 \\
6\end{array}$ & 16.80 & \begin{tabular}{|l}
$(15.20$ \\
$-18.40)$ \\
\end{tabular} & \\
\hline $\begin{array}{l}\text { Bone+Lung+ } \\
\text { Liver }\end{array}$ & 13 & $\begin{array}{l}(18.6 \\
\%)\end{array}$ & 8 & $\begin{array}{l}(61.5 \\
\%)\end{array}$ & 5 & $\begin{array}{l}(38.5 \\
\%)\end{array}$ & & 5 & $\begin{array}{l}38.5 \\
\%)\end{array}$ & 8 & $\begin{array}{l}(61.5 \\
\%)\end{array}$ & & $\begin{array}{l}13.4 \\
2\end{array}$ & $\begin{array}{l}+1.8 \\
4\end{array}$ & 13.50 & \begin{tabular}{|l|}
$(9.80$ \\
$16.20)$ \\
\end{tabular} & \\
\hline $\begin{array}{l}\text { Bone } \\
\text { metastasis }\end{array}$ & & & & & & & & & & & & & & & & & \\
\hline
\end{tabular}




\begin{tabular}{|c|c|c|c|c|c|c|c|c|c|c|c|c|c|c|c|c|c|}
\hline Absent & 30 & $\begin{array}{l}(42.9 \\
\%)\end{array}$ & 25 & $\begin{array}{l}83.3 \\
\%)\end{array}$ & 5 & $\begin{array}{l}(16.7 \\
\%)\end{array}$ & \multirow[t]{2}{*}{$\begin{array}{l}0.01 \\
3 \dagger\end{array}$} & \multicolumn{2}{|c|}{\begin{tabular}{l|l}
7 & $(23.3$ \\
& $\%)$
\end{tabular}} & \multicolumn{2}{|c|}{\begin{tabular}{c|c}
23 & $(76.7$ \\
$\%)$
\end{tabular}} & \multirow[t]{2}{*}{$\begin{array}{l}.14 \\
2 \ddagger\end{array}$} & $\begin{array}{l}14.8 \\
9\end{array}$ & \multicolumn{2}{|c|}{\begin{tabular}{|l|l|} 
\pm 2.6 & 14.80 \\
5 &
\end{tabular}} & $\begin{array}{l}(9.50 \\
- \\
19.30)\end{array}$ & \multirow{2}{*}{$\begin{array}{l}<0.00 \\
1 * \\
\end{array}$} \\
\hline Present & 40 & $\begin{array}{l}57.1 \\
\%)\end{array}$ & 22 & $(55 \%)$ & 18 & $(45 \%)$ & & $\begin{array}{l}1 \\
6\end{array}$ & $(40 \%)$ & 24 & $(60 \%)$ & & $\begin{array}{l}12.0 \\
5\end{array}$ & \pm 1.9 & 12 & $\begin{array}{l}(8.20 \\
- \\
16.20)\end{array}$ & \\
\hline $\begin{array}{l}\text { Brain } \\
\text { metastasis }\end{array}$ & & & & & & & & & & & & & & & & & \\
\hline Absent & 63 & $(90 \%)$ & 43 & $\left(\begin{array}{l}68.3 \\
\%)\end{array}\right.$ & 20 & $\begin{array}{l}(31.7 \\
\%)\end{array}$ & $\begin{array}{l}0.67 \\
6 \ddagger\end{array}$ & $\begin{array}{l}1 \\
6\end{array}$ & $(27 \%)$ & 46 & $(73 \%)$ & $\begin{array}{l}0.00 \\
4 \ddagger\end{array}$ & 13 & \begin{tabular}{|l|}
2.5 \\
5
\end{tabular} & 12.90 & $\begin{array}{l}(8.20 \\
(19.30)\end{array}$ & $\begin{array}{l}0.009 \\
*\end{array}$ \\
\hline Present & 7 & $(10 \%)$ & 4 & $\begin{array}{l}(57.1 \\
\%)\end{array}$ & 3 & $\begin{array}{l}(42.9 \\
\%)\end{array}$ & & 6 & $\begin{array}{l}85.7 \\
\%)\end{array}$ & 1 & $\begin{array}{l}(14.3 \\
\%)\end{array}$ & & $\begin{array}{l}15.7 \\
2\end{array}$ & $\begin{array}{l}+2.5 \\
0\end{array}$ & 15.30 & $\begin{array}{l}(11.80 \\
19.20)\end{array}$ & \\
\hline $\begin{array}{l}\text { Lung } \\
\text { metastasis }\end{array}$ & & & & & & & & & & & & & & & & & \\
\hline Absent & 39 & $\begin{array}{l}(55.7 \\
\%)\end{array}$ & 23 & $\mid(59 \%)$ & 16 & $(41 \%)$ & $\begin{array}{l}0.10 \\
3 \$\end{array}$ & $\begin{array}{l}1 \\
7\end{array}$ & $\begin{array}{l}(43.6 \\
\%)\end{array}$ & 22 & $\begin{array}{l}(56.4 \\
\%)\end{array}$ & $\begin{array}{l}0.03 \\
2 \ddagger\end{array}$ & $\begin{array}{l}12.9 \\
6\end{array}$ & & 12.30 & $\begin{array}{l}(8.20 \\
(19.30)\end{array}$ & $0.953^{\circ}$ \\
\hline Present & 31 & $\begin{array}{l}(44.3 \\
\%)\end{array}$ & 24 & $\begin{array}{l}(77.4 \\
\%)\end{array}$ & 7 & $\begin{array}{l}(22.6 \\
\%)\end{array}$ & & 6 & $\begin{array}{l}(19.4 \\
\%)\end{array}$ & 25 & $\begin{array}{l}(80.6 \\
\%)\end{array}$ & & $\begin{array}{l}13.6 \\
5\end{array}$ & \begin{tabular}{|l|}
2.0 \\
9
\end{tabular} & 13.80 & $\begin{array}{l}(9.50 \\
18.40)\end{array}$ & \\
\hline $\begin{array}{l}\text { Liver } \\
\text { metastasis }\end{array}$ & & & & & & & & & & & & & & & & & \\
\hline Absent & 50 & $\begin{array}{l}(71.4 \\
\%)\end{array}$ & 32 & $(64 \%)$ & 18 & $(36 \%)$ & $\begin{array}{l}0.37 \\
6 \$\end{array}$ & $\begin{array}{l}1 \\
8\end{array}$ & $(36 \%)$ & 32 & $(64 \%)$ & $\begin{array}{l}0.37 \\
6 \ddagger\end{array}$ & $\begin{array}{l}12.6 \\
6\end{array}$ & \pm 2.4 & 12.40 & $\begin{array}{l}(8.20 \\
(19.20)\end{array}$ & $\begin{array}{l}0.002 \\
* \\
*\end{array}$ \\
\hline Present & 20 & $\begin{array}{l}(28.6 \\
\%)\end{array}$ & 15 & $(75 \%)$ & 5 & $(25 \%)$ & & 5 & $(25 \%)$ & 15 & $(75 \%)$ & & $\begin{array}{l}14.7 \\
9\end{array}$ & \begin{tabular}{|l|}
2.6 \\
4
\end{tabular} & 14.50 & $\begin{array}{l}(9.80 \\
(19.30) \\
\end{array}$ & \\
\hline
\end{tabular}

Continuous variables were expressed as mean \pm SD \& median (range); categorical variables were expressed as number(percentage); *Independent samples Student's test for two groups and One Way ANOVA test for more than two groups; • Mann Whitney U test for two groups and Kraskall Wallis H test for more than two groups; $\$$ Chi-square test; $\S$ Chi-square test for trend; $\mathrm{p}<0.05$ is significant.

Effect of plasma S100p level, (bax and Bcl2) expression on treatment outcome in metastatic breast cancer patients: The implication of plasma S100P levels on the prognosis of our $70 \mathrm{MBC}$ patients was studied in our trial. Our patients were undergone follow up for 3 years (the median follow up period was 19 month). The cut off points of plasma S100P was $7.6 \mathrm{ng} / \mathrm{ml}$, which indicates that the values above it are high but the values below it are low.

Evaluation of the effect of the markers on treatment outcome (response to treatment, occurance of progression and mortality ) was done, which reveals significant correlation between plasma S100P level with each one of the following; response, progression, and mortality ( $\mathrm{P}<0.001$ for each ) (Table 4). Similarly , Bcl2 expression is significantly correlated with response ( $\mathrm{P}<0.001)$, progression ( $\mathrm{P} 0.004)$ and mortality ( $\mathrm{P} 0.01)$ (Table 4 ). However, bax expression bax was not significantly correlated with response $(P=0.78)$, progression $(P=0.80)$ or mortality $(P=0.80)$. 
Table (4): immunohistochemical staining for( $\mathrm{bcl} 2$, Bax) and plasmaS100p level on treatment outcome and survival in metastatic breast cancer patients $(\mathrm{N}=70)$.

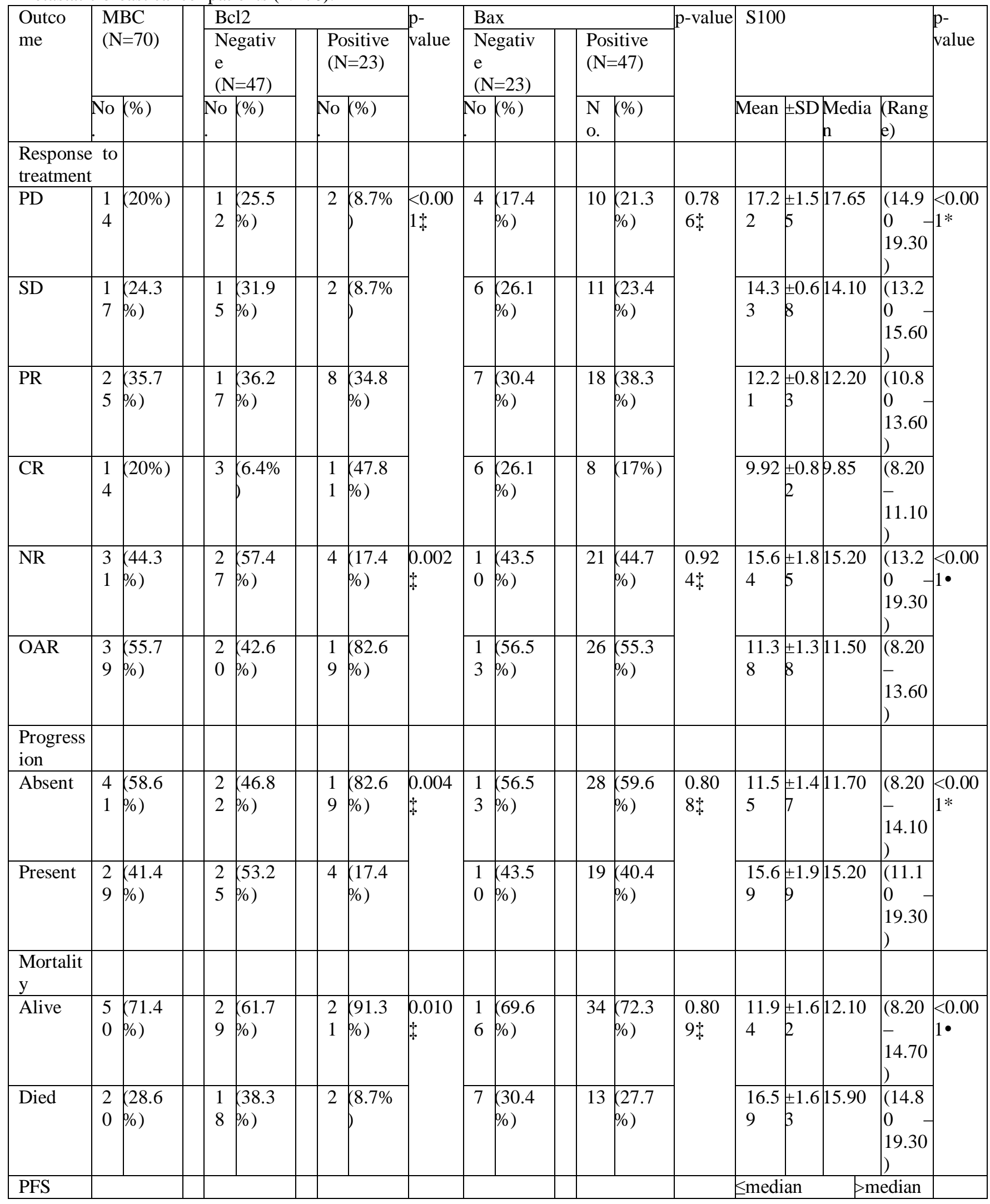




\begin{tabular}{|c|c|c|c|c|c|c|c|c|c|c|}
\hline & & & & & & & & $(\mathrm{N}=36)$ & \multirow[b]{2}{*}{$\begin{array}{l}(\mathrm{N}=34) \\
9.94 \\
\text { month } \\
(7.72- \\
12.15)\end{array}$} & \\
\hline $\begin{array}{l}\text { Mean } \\
\text { (month) } \\
\text { (95\%CI } \\
\text { ) }\end{array}$ & $\begin{array}{l}24.14 \\
\text { month } \\
(20.81- \\
27.46)\end{array}$ & $\begin{array}{l}19.69 \\
\text { month } \\
(15.84- \\
23.54)\end{array}$ & $\begin{array}{l}30.96 \\
\text { month } \\
(26.44- \\
35.47)\end{array}$ & \multirow[t]{4}{*}{$\begin{array}{l}0.005 \\
\dagger \dagger\end{array}$} & \multirow{2}{*}{\begin{tabular}{|l}
19.78 \\
month \\
$(15.42-$ \\
$24.14)$ \\
$60.9 \%$ \\
\end{tabular}} & \multirow{2}{*}{$\begin{array}{l}24.34 \\
\text { month } \\
(20.27- \\
28.41) \\
63.6 \%\end{array}$} & \multirow[t]{4}{*}{$\begin{array}{l}0.86 \\
0 \dagger\end{array}$} & $\begin{array}{l}35.36 \text { month } \\
(34.14-36.59)\end{array}$ & & \multirow[t]{4}{*}{$\begin{array}{l}<0.00 \\
1 \dagger\end{array}$} \\
\hline $\begin{array}{l}1 \text { year } \\
\text { PFS } \\
(\%)\end{array}$ & $62.7 \%$ & $52.9 \%$ & $82.6 \%$ & & & & & $100 \%$ & $22.4 \%$ & \\
\hline $\begin{array}{l}2 \text { years } \\
\text { PFS } \\
(\%)\end{array}$ & $57.9 \%$ & $44.8 \%$ & $82.6 \%$ & & $56.2 \%$ & $58.7 \%$ & & $96.9 \%$ & $13.5 \%$ & \\
\hline $\begin{array}{l}3 \text { years } \\
\text { PFS } \\
(\%)\end{array}$ & $57.9 \%$ & -- & $82.6 \%$ & & -- & -- & & $96.9 \%$ & -- & \\
\hline $\mathrm{OS}$ & & & & & & & & & & \\
\hline $\begin{array}{l}\text { Mean } \\
\text { (month) } \\
\text { (95\%CI } \\
\text { ) }\end{array}$ & $\begin{array}{l}30.75 \\
\text { month } \\
(27.68- \\
33.82)\end{array}$ & $\begin{array}{l}27.38 \\
\text { month } \\
(23.51- \\
31.25)\end{array}$ & $\begin{array}{l}36.30 \\
\text { month } \\
(32.72- \\
39.89)\end{array}$ & $\begin{array}{l}0.014 \\
\dagger\end{array}$ & $\begin{array}{l}26.34 \\
\text { month } \\
(22.18- \\
30.50)\end{array}$ & $\begin{array}{l}30.93 \\
(27.17- \\
34.68)\end{array}$ & $\begin{array}{l}0.90 \\
3 \dagger\end{array}$ & 36 month & $\begin{array}{l}16.56 \\
\text { month } \\
(13.86- \\
19.25)\end{array}$ & $\begin{array}{l}<0.00 \\
1 \dagger\end{array}$ \\
\hline $\begin{array}{l}1 \quad \text { year } \\
\text { OS }(\%)\end{array}$ & $84.3 \%$ & $80.9 \%$ & $91.3 \%$ & & $86.9 \%$ & $80.9 \%$ & & $100 \%$ & $67.7 \%$ & \\
\hline $\begin{array}{l}2 \text { years } \\
\text { OS }(\%)\end{array}$ & $70.9 \%$ & $60.7 \%$ & $91.3 \%$ & & $69.1 \%$ & $71.9 \%$ & & $100 \%$ & $37.8 \%$ & \\
\hline $\begin{array}{l}3 \text { years } \\
\text { OS }(\%)\end{array}$ & $70.9 \%$ & $60.7 \%$ & $91.3 \%$ & & -- & $71.9 \%$ & & $100 \%$ & -- & \\
\hline
\end{tabular}

Continuous variables were expressed as mean $(95 \% \mathrm{CI})$; categorical variables were expressed as number(percentage); *Independent samples Student's test for two groups and One Way ANOVA test for more than two groups; • Mann Whitney $\mathrm{U}$ test for two groups and Kraskall Wallis $\mathrm{H}$ test for more than two groups; $\$$ Chi-square test; $\dagger$ Log rank test; $p<0.05$ is significant.

Effect of plasma S100p level, (bax and Bcl2) expression on Survival in metastatic breast cancer patients:

Mean PFS was 24.14 months, 3 y PFS was $57.9 \%$. Mean OS was 30.75 month, 3y OS was $70.9 \%$. The association between plasma S100P levels and PFS was assessed in our MBC patients. As illustrated in Kaplan-Meier curve, MBC patients with lower plasma S100P level had significantlylonger PFS in comparison to those who had higher plasma S100P levels (the mean PFS 35.36 months VS 9.94 months respectively, $\mathrm{P}=0.000$, Fig.1B) .Moreover, patients with positive bcl2 expressions had significantly longer PFS when compared to those who had negative bcl2 expression (the mean PFS time was 30.96 months VS 19.69 months respectively, $\mathrm{P}=0.005$, Fig.1C). While, there is insignificant increase in the mean PFS time for the patients who had BAX positive expression versus BAX negative patients (the mean PFS time was 24.34 months VS 19.78 months respectively, $\mathrm{P}=0.860$, Fig.1D).

Overall survival $(\mathrm{OS})$ in the studied MBC patients $(\mathrm{N}=70)$ showed that, patients who had low plasma S100P levels had significantly better OS than those who had high plasma S100P levels (the mean OS was 36.3 months VS 16.56 months respectively, $\mathrm{P}=0.000$, Fig.2B), as well, patients with positive bcl2 expressions had significantly longer OS when compared to those who had negative bcl2 expression (the mean OS was 36.30 months VS 27.38 months respectively, $\mathrm{P}=0.014$, Fig.2C). Whereas there is insignificant increase in the mean OS for the patients with bax positive expression versus bax negative patients (the mean OS was 30.93 months VS 26.34 months respectively, P=0.903, Fig.2D). 

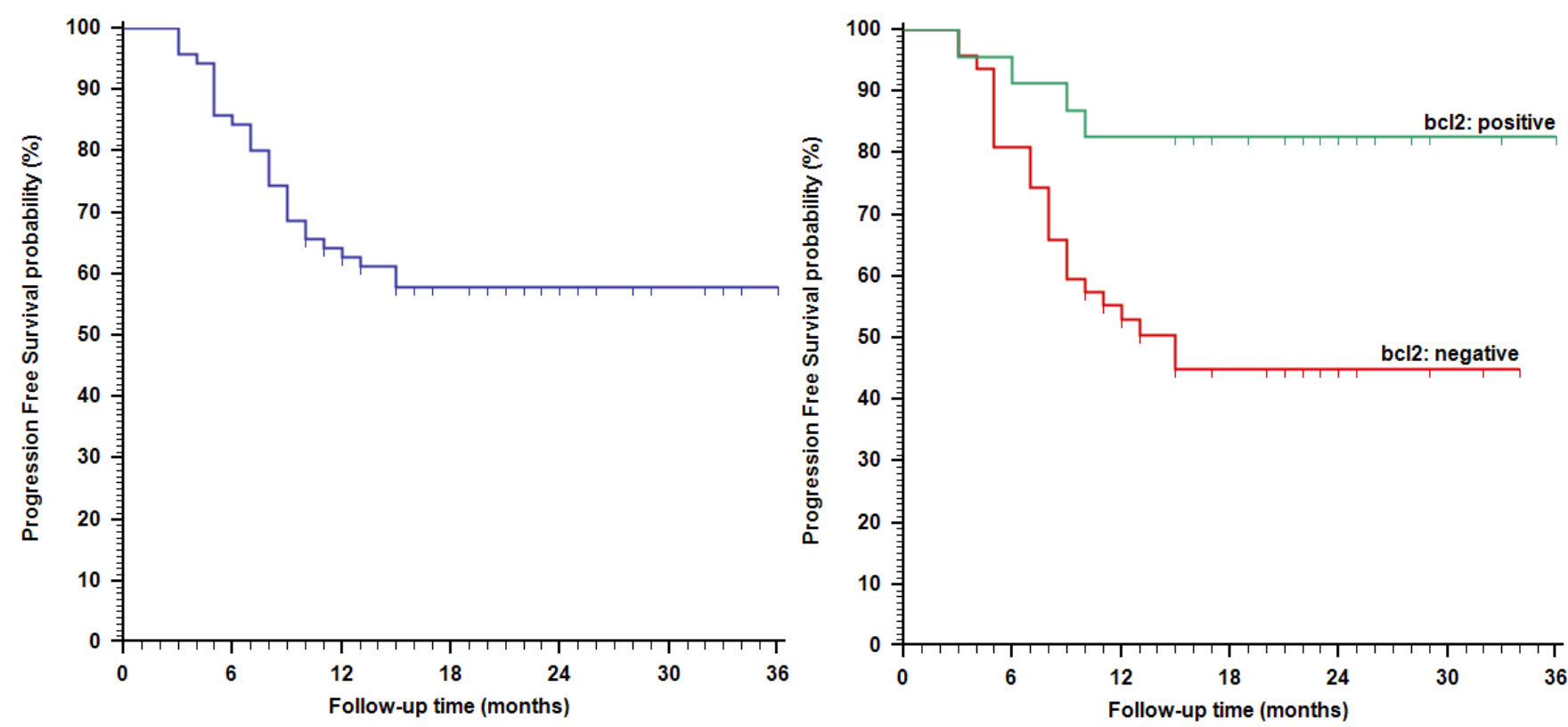

(A)

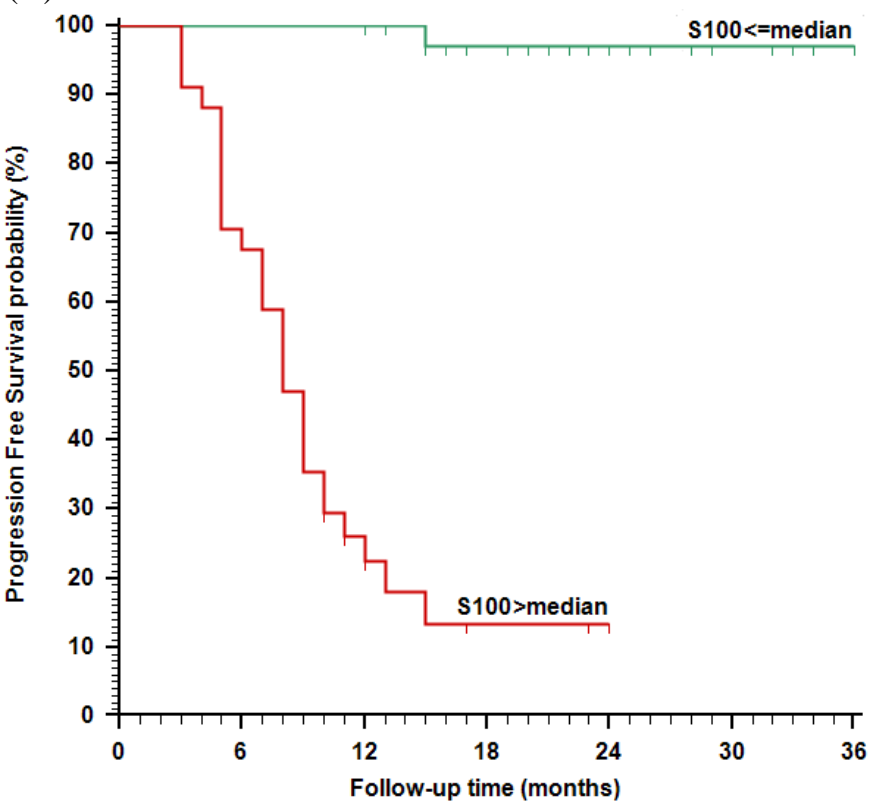

(C)

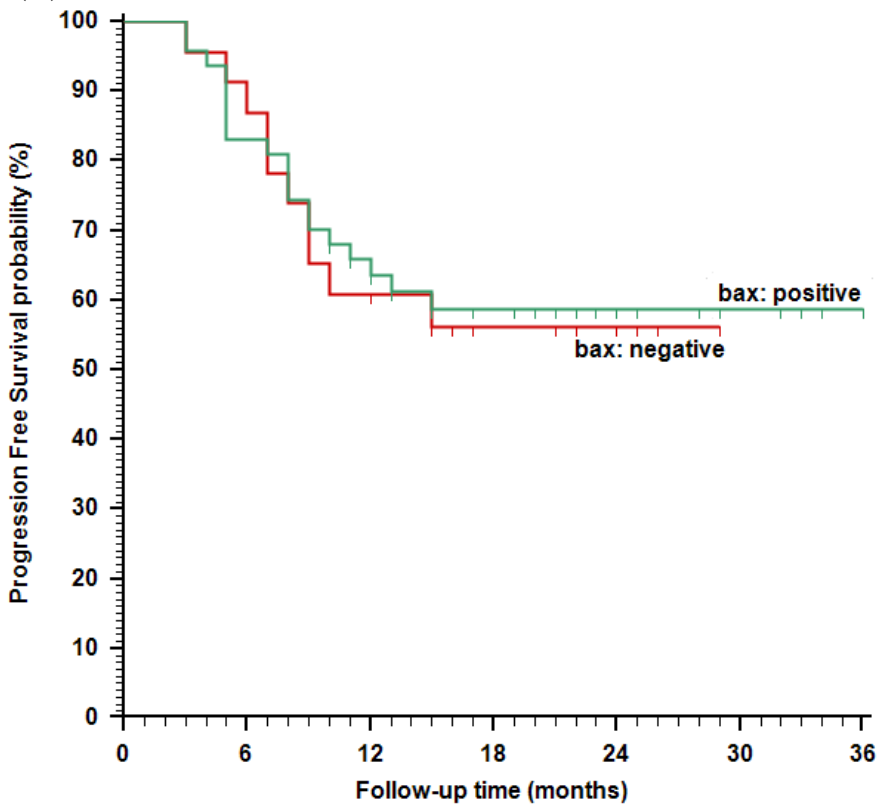

(D)

(B)

Fig. (1): Kaplan Meier plot of progression free survival (PFS) in the studied MBC patients ( $N=70$ ): (A) All studied patients; (B) Stratified by S100; (C) Stratified by bcl2; (D) Stratified by bax. 

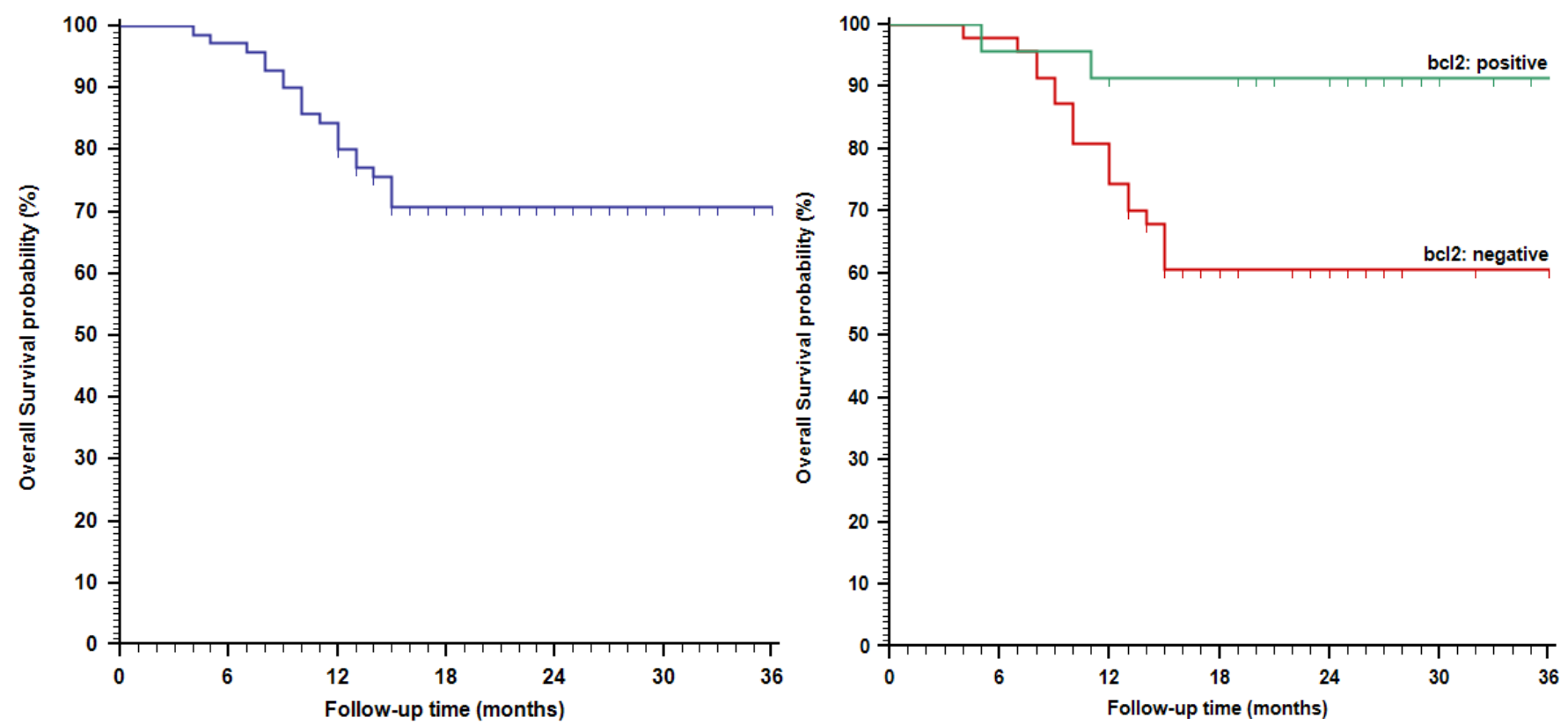

(A)

(C)
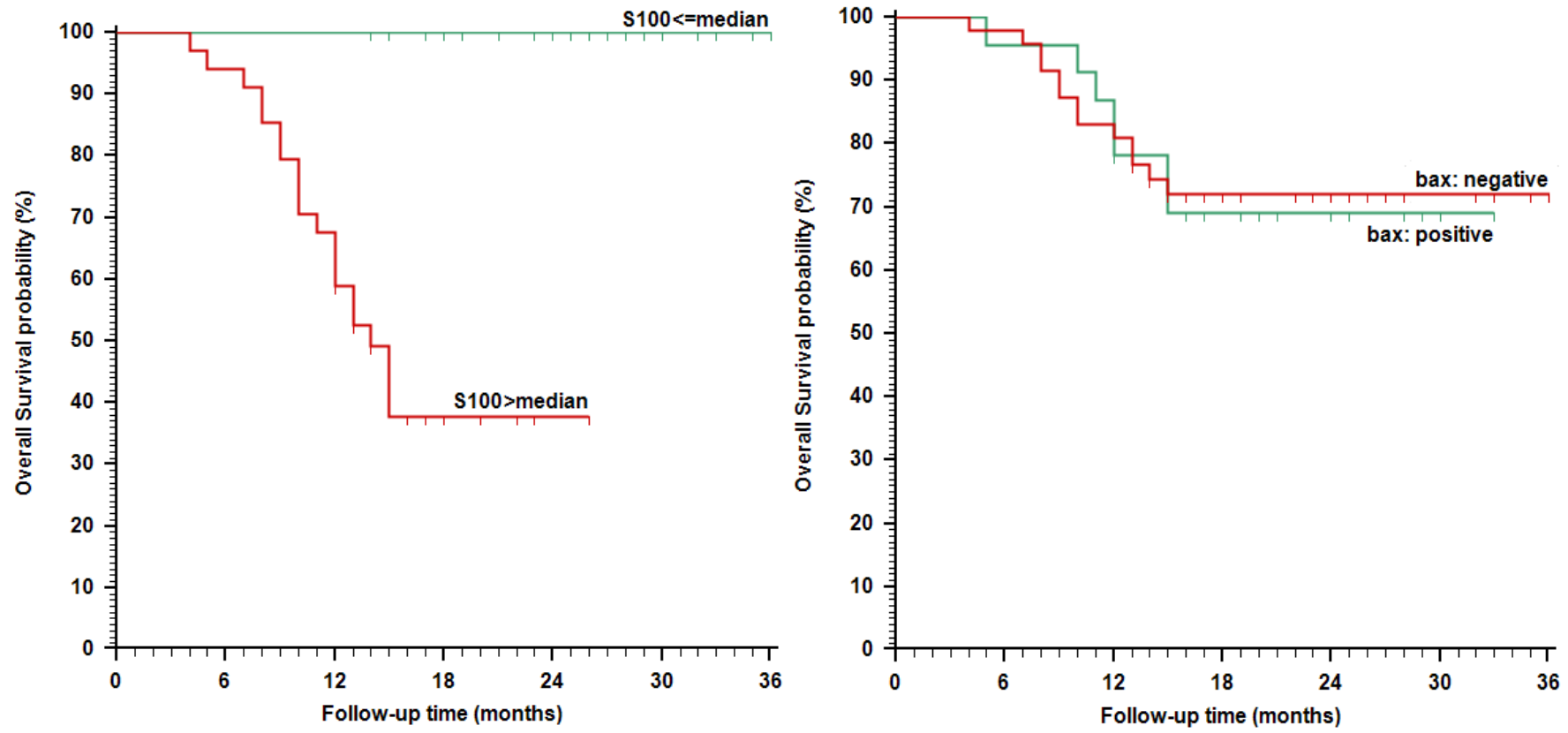

(B)

(D)

Fig. (2): Kaplan Meier plot of overall survival (OS) in the studied MBC patients (N=70): (A) All studied patients; (B) Stratified by S100; (C) Stratified by bc12; (D) Stratified by bax. 

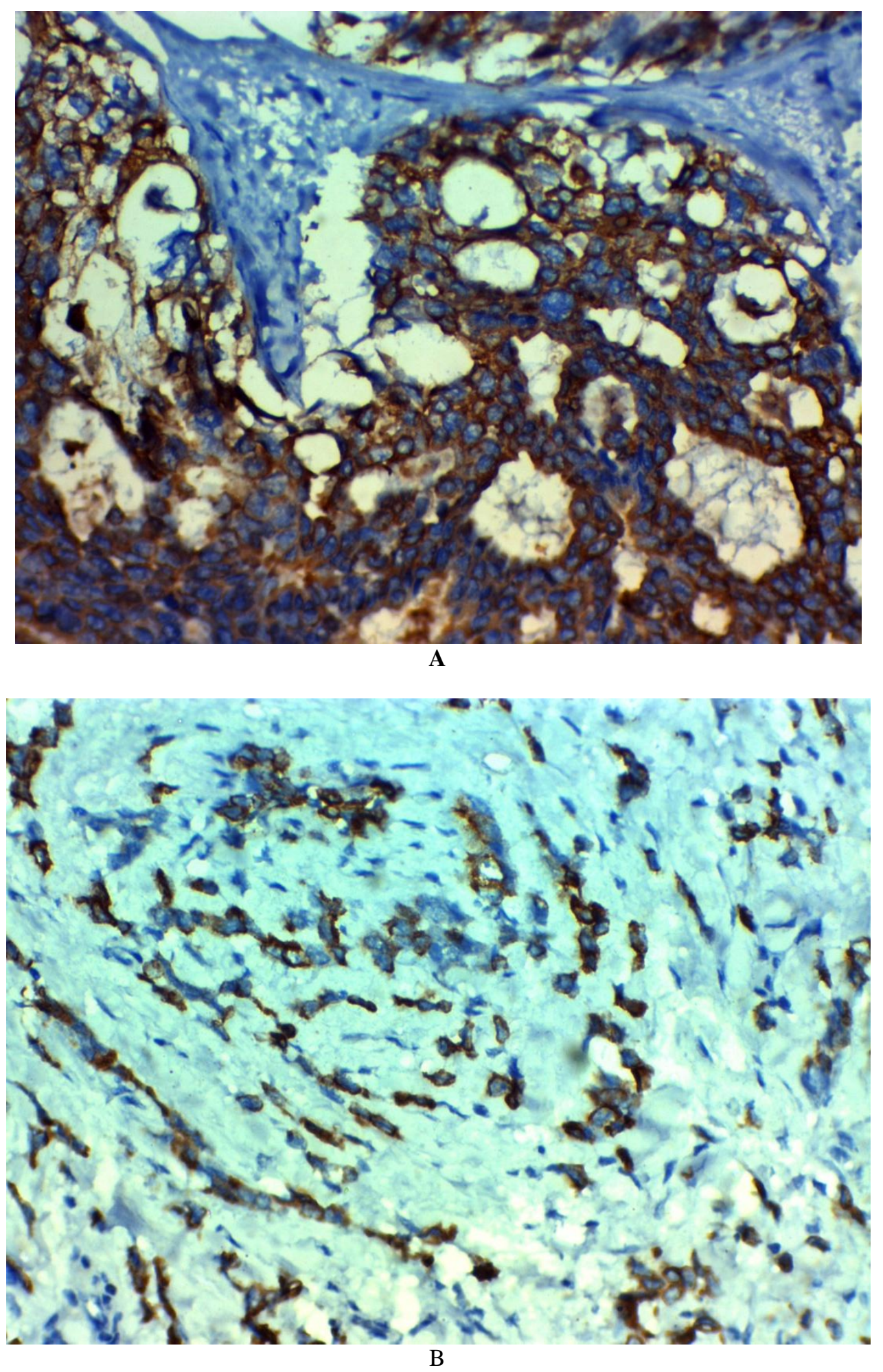


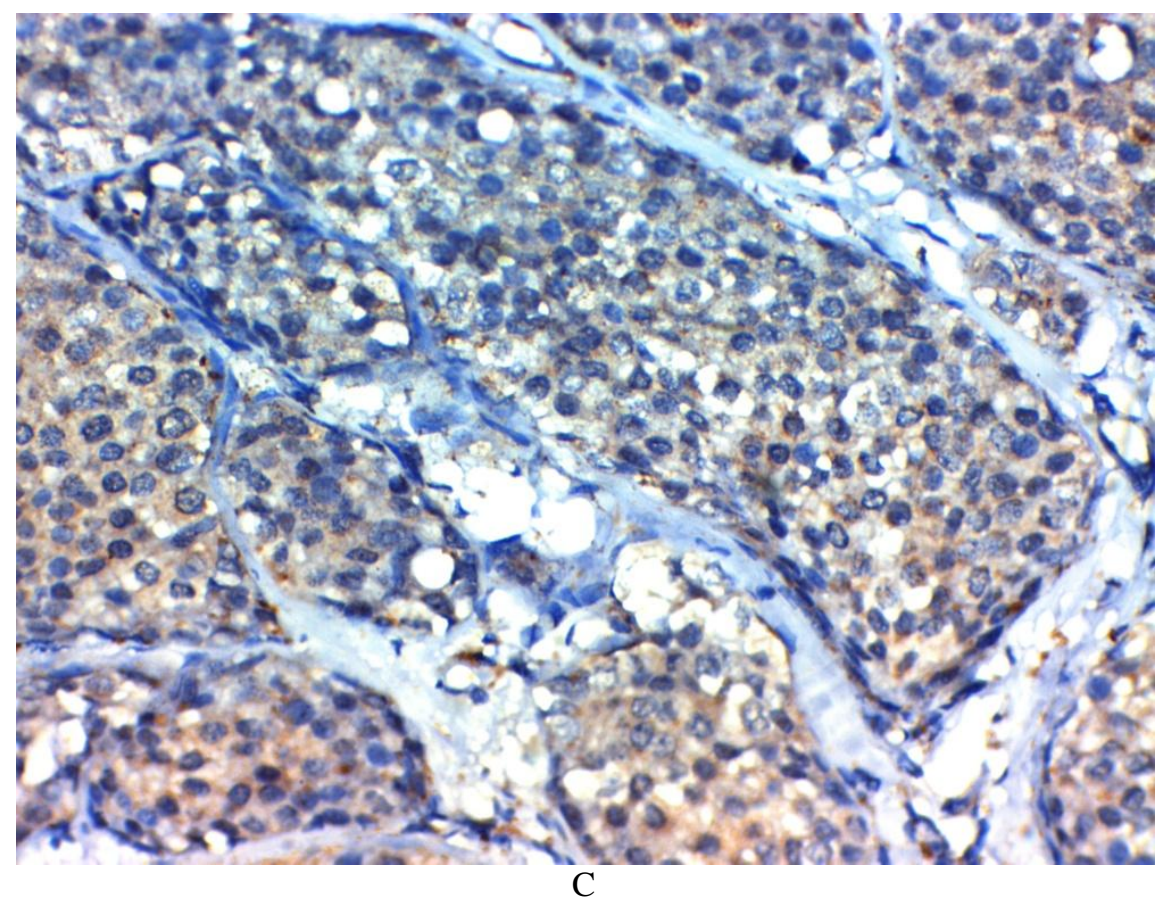

Figure1. Immunohistochemical staining of Bax in infiltrating carcinoma of the breast: (A) High Immunohistochemical expression in the cytoplasm of high grade infiltrating duct carcinoma of the breast x400. (B) High Immunohistochemical expression in the cytoplasm of infiltrating lobular carcinoma of the breast stage IV ; (C) Low Immunohistochemical expression in the cytoplasm of low grade infiltrating duct carcinoma of the breast $\mathrm{x} 400$.

Note: High Bax immunohistochemical expression in high grade\& stage carcinoma of the breast and low expression in low grade\& stage carcinoma of the breast Magnification: A, B\& C the original magnification was $\times 400$

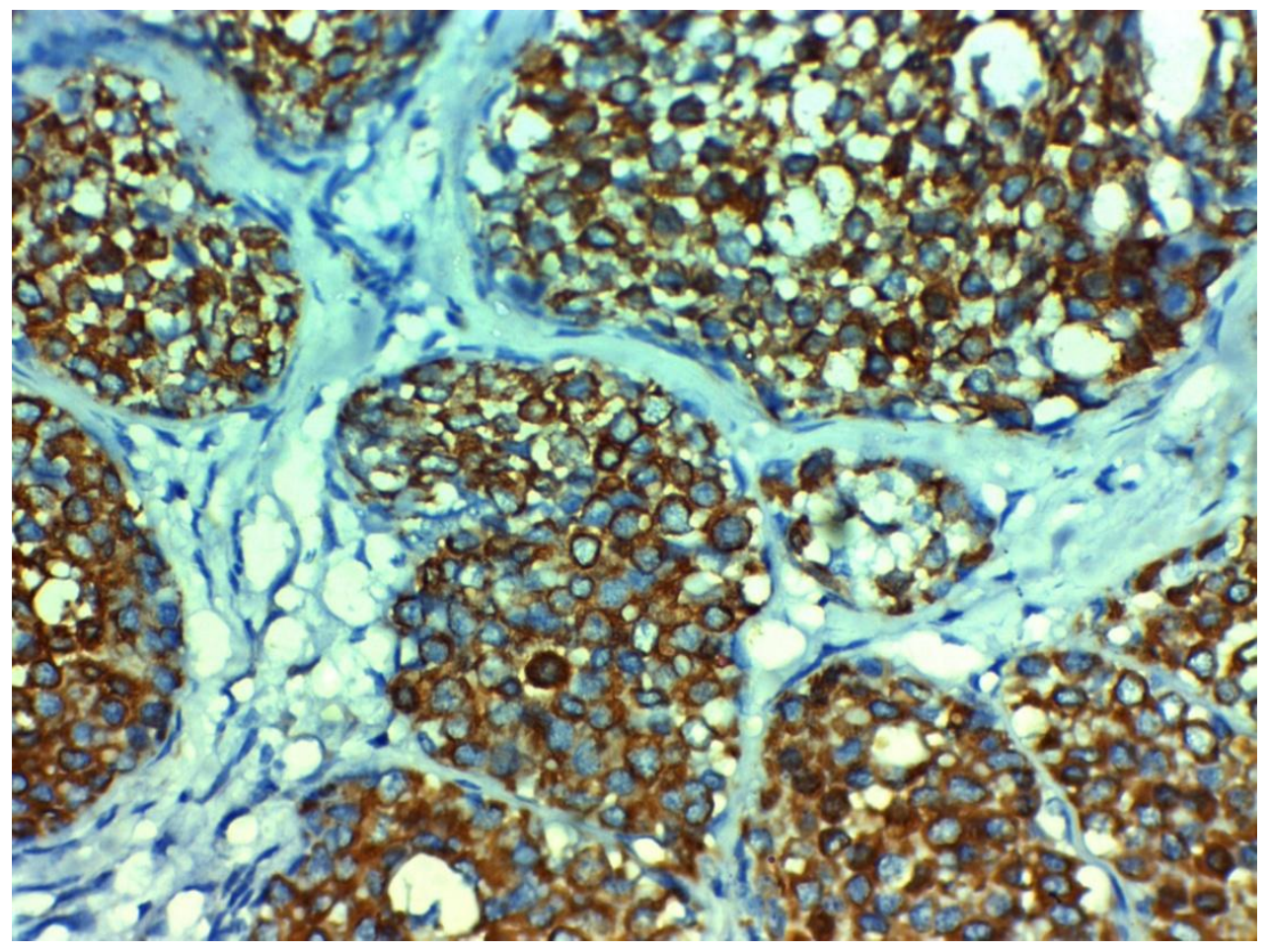



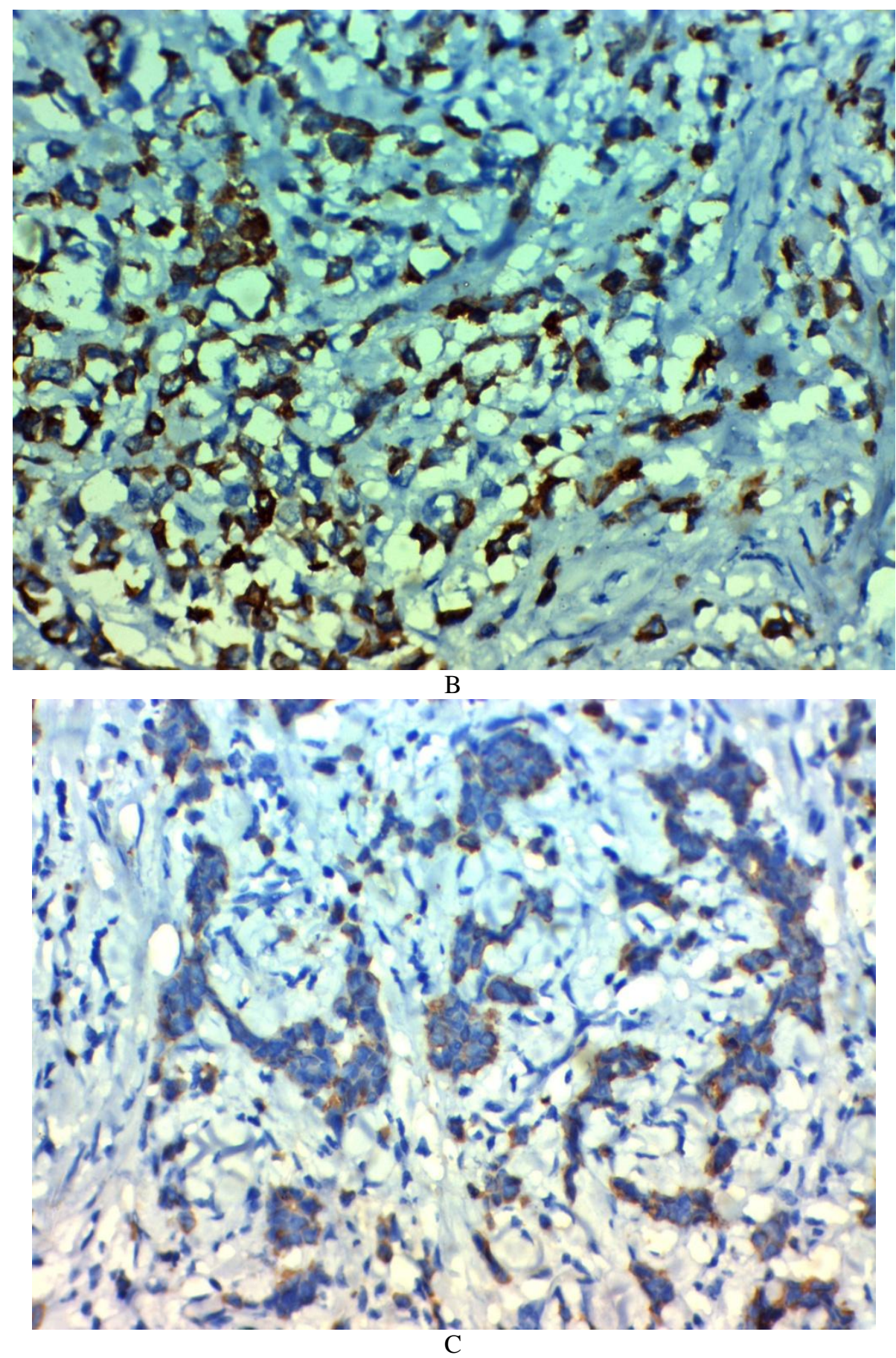

Figure2. Immunohistochemical staining of Bcl-2 in carcinoma of the breast: (A) High Immunohistochemical expression in the cytoplasm of low grade infiltrating duct carcinoma of the breast $x$ 400. (B) High Immunohistochemical expression in the cytoplasm of infiltrating lobular carcinoma of the breast ; (C) Low Immunohistochemical expression in the cytoplasm of high grade infiltrating duct carcinoma of the breast $x 400$.

Note: High Bax immunohistochemical expression in high grade\& stage carcinoma of the breast and low expression in low grade\& stage carcinoma of the breast Magnification: A, B\& C the original magnification was $\times 400$ 


\section{Discussion:-}

Breast cancer is the most common diagnosed cancer all over the world and the main cause of cancer-related death among females (18). In addition, up to 30 percent of women with early-stage breast cancer at time of diagnosis will develop distant metastatic disease. Although metastatic breast cancer (MBC) is not curable; the introduction of newer systemic therapies leads to survival improvement (19-21).

In our study; we followed the patients for 3 years (the median follow up period was 19 month). The cut off points of plasma S100P was $7.6 \mathrm{ng} / \mathrm{ml}$, which indicates that the values above it are highbut the values below it are low . S100P level in metastatic breast cancer patients are higher than PBC and healthy tissue. Elevation of Serum S100P level in metastatic breast cancer patients showed significant correlation with capsular invasion, bcl2 $(\mathrm{P}=0.002$ and $<0.001$ respectively), increased number of metastasis, metastatic site $(\mathrm{P}<0.001$ for both $) ;\{$ presence of liver metastasis $\mathrm{P}=0.002$, brain metastasis $\mathrm{P}=0.009$ and bone metastasis $\mathrm{P}<0.001\}$.

There is a significant correlation between plasma S100P level with response, progression, and mortality $(\mathrm{P}<0.001$ for each). MBC patients with lower plasma S100P level had longer but non-significant PFS time in comparison to those who had higher plasma S100P levels (the mean PFS time 35.36 months VS 9.94 months respectively, log-rank test P=0.000) Patients who had low plasma S100P levels had better but non-significant OS than those who had high plasma S100P levels (the mean OS time was 36.3 months VS 16.56 months respectively, log-rank test $\mathrm{P}=0.000$ )

The small, calcium-binding protein S100P has gained the attention of researchers from different scientific fields due to its potential roles in both healthy and neoplastic tissues(22). S100P is a member of the large family of S100 calcium-binding proteins that mediate $\mathrm{Ca}^{2+}$ dependent signal transduction pathways (23). Its expression has been found frequently and at high levels, in a variety of different tumor types in addition to its role in chemoresistance $(\mathbf{3}, \mathbf{4})$. S100P could potentially serve as diagnostic marker, prognostic, predictive indicator and therapy target for different carcinomas through its inhibition or inhibition of its targets, or its interactions which result in a decrease of cellular motility and metastatic potential(7, 24).

S100P plays an important role in oncogenesis (tumor cell proliferation, differentiation, apoptosis, weakening of cell: cell adhesion contacts, stimulation of cell motility and invasion and metastasis $\quad(\mathbf{2 5}, \mathbf{2 6})$.

S100 $\mathrm{P}$ is expressed in tumor tissue and absent in most healthy tissues, so it was evaluated as a novel biomarker for detection of several cancers by using immunohistochemistry approaches (24). But in our study we used ELISA as a new modality for measurement of S100 P.

Indeed, anti-S100P antibodies have shown promising results (in vitro and in vivo) as single agents and in combination with other chemotherapeutic agents, such as gemcitabine in pancreatic cancer (3). So blocking S100P function is expected to improve responses to chemotherapeutic agents. However, this needs further investigations because there are other reports showed that overexpression of S100P (in vitro) led to sensitization of cancer cells to carboplatin, paclitaxel (27) and oxaliplatin (28) in ovarian and gastric cancer cells respectively. Despite this, S100P still represents a potentially very effective anti-cancer target, at least for in some cancer types, and further development of anti-S100P specific therapies will likely prove to be a fruitful and productive field of investigation (29).

In breast cancer, S100P expression is associated with immortalization of neoplastic cells and aggressive tumor behavior, indicating that this protein may have adverse prognostic value and poor survival in breast cancer patients (30).

Univariate and multivariate analyses in early breast cancer patients (stage II) showed that higher expression of nuclear S100P (S100Pn) was observed in cases of a shorter overall survival and disease-free time. No relationship could be documented between expression of S100P and sensitivity of breast cancer cells to cytostatic drugs. The preliminary data indicated that, this protein might become a therapy target and warrants further studies with respect to its prognostic, predictive and potentially therapeutic value.(30).

Plasma S100P levels were measured in 381 women, including 60 healthy controls, 48 primary breast cancer patients (PBC) patients, and 273 metastatic breast cancer (MBC) with correlation between increased its level and MBC . In addition, assessment of prognostic value of S100P with enumeration of CTC and clinicopathological factors were done .The follow up period was 3.5 years. They found that the plasma S100P cut off point was $7 \mathrm{ng} / \mathrm{ml}$ and also there is association between high plasma S100P level $(>7 \mathrm{ng} / \mathrm{mL})$ and poor prognosis of MBC patients (median progression-free survival time: 5.0 vs. 8.7 months, log-rank test $p<0.001$; median overall survival time: 22.5 vs. 31.6 months, $\log$-rank test 
$\mathrm{p}<0.001)$. The plasma S100P level added additional prognostic relevance to the prognostication model with clinicopathological factors and CTC enumeration. Furthermore, The examination of the value of plasma S100P levels as treatment monitoring marker was done and revealed, its significant reduction after treatment, This indicates its value in evaluation of treatment outcome. They concluded that plasma S100P level is a simple and cost-effective marker for the prognosis of metastatic breast cancer (4).

The results of this trial are consistent with our result where, the cut off points of plasma S100P was $7.6 \mathrm{ng} / \mathrm{ml}$, which indicate that the values above it are highbut the values below it are low. S100P level in metastatic breast cancer patients are higher than PBC and healthy tissue. There is significant correlation between plasma S100P level with response, progression, and mortality $(\mathrm{P}<0.001$ for each). MBC patients with lower plasma S100P level had significant longer PFS time in comparison to those who had higher plasma S100P levels (the mean PFS time 35.36 months VS 9.94 months respectively, log-rank test $\mathrm{P}=0.000$ ) Patients who had low plasma S100P levels had significant better OS than those who had high plasma S100P levels (the mean OS time was 36.3 months VS 16.56 months respectively, log-rank test $\mathrm{P}=0.000$ ) .

There are many theories about the lack of activity of anti-cancer drugs in breast cancer. The disruption of the apoptotic pathways may be one of reasons. We therefore decided to assess the expression of those factors involved in apoptosis in the normal mammary gland, benign mammary dysplasia and primary cancer. The most significant findings of our study are that, Bcl2 positive protein expressions in breast cancer tissues were lower than those in the healthy and adjacent breast tissues. Moreover, its expression in all breast cancer patients was significantly correlated with good clinic pathological parameters like low grade and stage $(\mathrm{P}<0.001)$ and low ki67 level $(\mathrm{P} 0.021)$. Also its positive expression in MBC patients was statistically significant with low ki67 level (P 0.044), molecular subtype (P 0.050), and low grade, T, N, Bax (P <0.001 for each of them) decreased number of metastasis $(\mathrm{P}=0.014)$ and presence of bone metastasis $(\mathrm{P}=0.013)$.

Bax positive protein expressions in breast cancer tissues were higher than that in the relatively healthy, adjacent breast tissues. Furthermore, its positive expression was significantly correlated with poor clinic pathological parameters like high grade $(\mathrm{P}=0.003)$ and stage $(\mathrm{P}<0.001)$, high ki67 level $(\mathrm{P}<0.004)$, positive $\mathrm{ER}(\mathrm{P}<0.010)$, $\mathrm{PR}(\mathrm{P}<0.023)$, Her-2 neu $(\mathrm{P}<0.002)$ and negative bcl2 level $(\mathrm{P}<0.001)$ in all breast cancer patients. However in $\mathrm{MBC}$ patients its positive expression is statistically significant with high grade $(\mathrm{P}=0.001)$, every one of molecular subtype, $\mathrm{T}, \mathrm{N}(\mathrm{P}<0.001$ for each), high ki67 level $(\mathrm{P}<0.002)$, and site of metastasis $(\mathrm{P}=0.003)$ brain $(\mathrm{P}=0.004)$, lung $(\mathrm{P}=0.032)$. However, bax expression is not significant with either response $(\mathrm{P}=0.78)$, progression $(\mathrm{P}=0.80)$ nor mortality $(\mathrm{P}=0.80)$. Similarly, $\mathrm{Bcl} 2$ expression is significantly correlated with response ( $\mathrm{P}<0.001$ ), progression ( P 0.004) and mortality ( P 0.01) Mean PFS was 24.14 months, 3 y PFS was $57.9 \%$. Mean OS was 30.75 month, 3y OAS was $70.9 \%$. Patients with positive bcl2 expressions had significantly longer PFS when compared to those who had negative bcl2 expression (the mean PFS time was 30.96 months VS 19.69 months respectively, log-rank test $\mathrm{P}=0.005$ ) .Meanwhile, there is insignificant increase in the mean PFS time for the patients who had Bax positive expression versus Bax negative patients (the mean PFS time was 24.34 months VS 19.78 months respectively, log-rank test $\mathrm{P}=0.860$ ). Patients with positive bcl2 expressions had significantly longer OS when compared to those who had negative bcl2 expression (the mean OS time was 36.30 months VS 27.38 months respectively, $\log$-rank test $\mathrm{P}=0.014)$. But, there is insignificant increase in the mean OS time for the patients who had Bax positive expression versus Bax negative patients (the mean OS time was 30.93 months VS 26.34 months respectively, log-rank test $\mathrm{P}=0.903)$

Liu et al., showed that a high apoptotic rate is associated with a high grade of tumor, large tumor size and with a shortened disease-free survivalperiod (31). In the study of Ioachim et al. (2000) (32) Bcl-2 protein was detected in $85.2 \%$ of benign hyperplastic lesions of the mammary gland and $40 \%$ of breast cancers. On the other hand, Bargou et al. (1995) (33) observed no difference with regard to Bcl-2 (and Bcl-XL) expression between normal breast epithelium and breast cancer tissue. Similarly to Bargou et al. (1995)(33), In the study by Gee et al., (1994)(34) Bcl-2 was detected in 70\% of breast cancers. It has also been shown that Bcl-2-positive patients had a better prognosis than Bcl-2-negative patients (35) .

Rochaix et al.(1999) (36) suggested that Bcl-2 and Bax expression were associated with a regulation of apoptosis in breast cancer.They found that Bcl-2 expression in tumours was associated with a better differentiation of the cancers (G1 $100 \%$ of Bcl-2-positive tumours, G2 - 81\%, G3 - 60\%), but there was no relationship between Bax and tumor grade .

In the study of Berardo et al. [1998](37)high Bcl-2 expression was associated with favourable prognostic factors such as ER positivity, low $S$ phase fraction, a lower number of positive lymph nodes and overall survival. Our findings confirm the results of Berardo et al. [1998](37) with regard to the favourable prognostic significance of Bcl-2 expression in breast cancer.Honma et al., 2015(38) Compared Bcl-2 expression with other clinicopathological factors ,Bcl-2 positivity was 
significantly correlated with smaller tumor size, lower grade, ER positivity, PR positivity, and HER2 negativity, in both groups, confirming Bcl-2's association with favorable prognostic factors.

Dawson et al 2010(39)revealed that BCL2 continues to be associated with favorable outcome. BCL2 belongs to a group of related proteins that are key regulators of apoptosis or programmed cell death (Cory et al, 2003)(40).

BCL2 protein expression in breast cancer is associated with an indolent phenotype of low-grade, slowly proliferating, ERp breast tumours (Silvestrini et al, 1994; Lipponen et al, 1995)(41,42). This 'paradoxical' favourable prognostic effect of BCL2 in breast cancer could be related to its non-apoptotic functions (43). Increased expression of BCL2 protein may also disrupt the balance with other members of the BCL2 family, including the expression of pro-apoptotic proteins (40) .The exact mechanism of differential BCL2 protein expression in breast cancer is complex. BCL2 is expressed in normal breast glandular epithelium and is known to be upregulated by oestrogen, possibly as a direct result of transcriptional induction (44)

Also no differences in treatment response were found in patients with early breast cancer related to Bax expression in the tumor cells (45). While Krajewski et al. detected that, MBC patients with low Bax expression had poorer response to treatment and shorter OS (46).

Pluta P, et al studied 62 breast cancer patients and control group of 11 breast fibroadenoma patients, bax expression was assessed by flow cytometer, bax expression was found in $82 \%$ of patients. Bax expression was lower in breast cancer patients than in controls, and this could be one of the mechanisms of apoptosis escaping by tumor cells (47).

Novel markers that could be used to save women from unnecessary cytotoxic adjuvant therapy are urgently needed and BCL2 provides valuable additional prognostic information to guide clinical decision making in this setting. In summaryour results proved that bcl 2 and bax are independent and powerful prognostic protein marker in breast cancer patients more than other prognostic factors.

Our results indicate that overexpression of pro-apoptotic proteins could contribute to an increase in cell turnover and breast cancer development and progression, but we suggest that further studies should be carried out with increased sample size to fully assess Bak expression in breast cancer progression.

Conclusion: S100P, BCL2 and bax are promising prognostic markers in breast cancer patients but we recommend further studies with large sample size to be done to increase statistical power of the results.

\section{Reference:-}

1. Siegel R, Ma J, Zou Z, et al (2014); Cancer statistics. CA: A Cancer J Clin. 64:9-29.

2. Amal S. Ibrahim, Hussein M. Khaled, et al (2014):- Cancer Incidence in Egypt: Results of the National PopulationBased Cancer Registry Program. Journal of Cancer Epidemiology, Volume, Article ID 437971, 18

3. Dakhel S, Padilla L, Ada $_{\mathrm{n}} \mathrm{J}$, et al (2014):-S100P antibody-mediated therapy as a new promising strategy for the treatment of pancreatic cancer. Citation: Oncogenesis 3, 92; doi:10.1038/oncsis.2014.7

4. Peng C , Chen H , Wallwiener M (2016):-Plasma S100P level as a novel prognostic marker of metastatic breast cancer.Breast Cancer Res Treat; 157(2):329-38.

5. Ohuchida K, Mizumoto K, Egami T, et al (2006). S100P is an early developmental marker of pancreatic carcinogenesis. Clin Cancer Res 12(18):5411-5416.

6. Li Y, St John MA, Zhou X,et al (2004):- Salivary transcriptome diagnostics for oral cancer detection. Clin Cancer Res 10(24):8442-8450.

7. Thompson CB (1995):- Apoptosis in the pathogenesis and treatment of disease. Science. 267:1456-1462

8. Wyllie AH (1987):- Apoptosis: cell death in tissue regulation. J Pathol ; 153:313-316.

9. Kerr JF, Winterford CM and Harmon BV (1994):- Apoptosis. Its significance in cancer and cancer therapy. Cancer. ; 73:2013-2026.

10. Meterissian SH (1997):- Apoptosis: its role in the progression of and chemotherapy for carcinoma. J Am Coll Surg, 184:658-666.

11. Cleary ML, Smith SD and Sklar J (1986):- Cloning and structural analysis of cDNAs for bcl-2 and a hybrid bcl2/immunoglobulin transcript resulting from the $\mathrm{t}(14 ; 18)$ translocation. Cell; 47:19-28.

12. Adams JM and Cory S (1998):-The Bcl-2 protein family: arbiters of cell survival. Science; 281:1322-1326.

13. Edge SB and Compton CC. (2010) :-The American Joint Committee on Cancer: the 7th edition of the AJCC cancer staging manual and the future of TNM.Ann Surg Oncol. Jun; 17(6):1471-4. doi: 10.1245/s10434-010-0985-4. 
14. Elston CW(2002) :- Pathological prognostic factors in breast cancer I. The value of histological grade in breast cancer: experience from a large study with long-term follow-up. Histopathology 1991, 19:403-410.

15. Hsu SM, Raine L and Fanger H (1981):- Use of avidin-biotin-peroxidase complex (ABC) in immunoperoxidase techniques: a comparison between $\mathrm{ABC}$ and unlabeled antibody (PAP) procedures. J HistochemCytochem ; 29:577580

16. Zhang G-J, Kimijima I, Abe R, et al(1997):-Correlation between the expression of apoptosis-related bcl-2 and p53 oncoprotein and the carcinogenesis and progression of breast cancer. Clin Cancer Res; 3:2329-35.

17. Veronesel S, Francesco A. Mauri I, et al(1998): Bax immunohistochemical expression in breast cancer.A study with long term follow up .Int. J. Cancer (Pred. Oncol.): 79, 13-18 .

18. Breast Cancer. http://www.cancerresearchuk.org/cancer-info/cancerstats/world/breast-cancer-world/ (Accessed on March 04, 2013).

19. Early Breast Cancer Trialists' Collaborative Group (EBCTCG) (2005):- Effects of chemotherapy and hormonal therapy for early breast cancer on recurrence and 15-year survival: an overview of the randomised trials. Lancet ; 365:1687.

20. Chia SK, Speers CH, D'yachkova Y, et al (2007):- The impact of new chemotherapeutic and hormone agents on survival in a population-based cohort of women with metastatic breast cancer. Cancer ; 110:973.

21. Dafni U, Grimani I, Xyrafas A, et al.(2010):- Fifteen-year trends in metastatic breast cancer survival in Greece. Breast Cancer Res Treat ; 119:621.

22. Prica F, RadonT, Cheng Y,et al (2016):- The life and works of S100P - from conception to cancer. . Am J Cancer Res. 2016; 6(2): 562-576.

23. Donato R (2001):- S100: a multigenic family of calcium-modulated proteins of the EF-hand type with intracellular and extracellular functional roles. Int J Biochem Cell Biol ;33:637-668.

24. Parkkila S, Pan PW, Ward A, et al (2008):- The calcium binding protein S100P in normal and malignant human tissues. BMC Clin Pathol ;8:2.

25. Ciocca DR and Calderwood SK (2005):- Heat shock proteins in cancer: diagnostic, prognostic, predictive, and treatment implications. Cell Stress Chaperones ;10:86-103.

26. Johnson M, Sharma M, Henderson BR (2009):- IQGAP1 regulation and roles in cancer. Cell Signal ;21:1471-1478.

27. Wang Q, He Z, Gao J, et al (2008):-. S100P sensitizes ovarian cancer cells to carboplatin and paclitaxel in vitro. Cancer Lett. ;272:277-284.

28. Zhao X, Bai Z, Wu P, et al (2013):-. S100P enhances the chemosensitivity of human gastric cancer cell lines.Cancer Biomark. ;13:1-10.

29. Tong XM, Lin XN, Song T, et al (2010):- Calcium-binding protein S100P is highly expressed during the implantation window in human endometrium. Fertil Steril; 94:1510-1518.

30. Maciejczyk A, Lacko A, Ekiert M,et al (2013):-Elevated nuclear S100P expression is associated with poor survival in early breast cancer patients. Histol Histopathol ;28:513-52476

31. Liu S, Edgerton SM, Moore DH, et al (2001) :- Measures of cell turnover (proliferation and apoptosis) and their association with survival in breast cancer. Clin Cancer Res, 7: 1716-1723

32. Ioachim EE, Malamou-Mitsi V, Kamina SA, et al(2000):-Immunohistochemical expression of Bcl-2 protein in breast lesions: correlation with Bax, p53, Rb, C-erbB-2, EGFR and proliferation indices. Anticancer Res, 20: 4221-4225.

33. Bargou RC, Daniel PT, Mapara MY, et al (1995) :-Expression of the bcl-2 gene family in normal and malignant breast tissue: low bax-alpha expression in tumor cells correlates with resistance towards apoptosis. Int J Cancer, 60: 854-859.

34. Gee JM, Robertson JF, Ellis IO, et al (1994) :-Immunocytochemical localization of Bcl-2 protein in human breast cancers and its relationship to a series of prognostic markers and response to endocrine therapy. Int J Cancer, 59: 619628.

35. Zhang GJ, Kimijima I, Abe R, et al (1998):- Apoptotic index correlates to bcl-2 and p53 protein expression, histological grade and prognosis in invasive breast cancers. Anticancer Res, 18: 1989-1998

36. Rochaix P, Krajewski S, Reed JC, et al(1999):- In vivo patterns of Bcl-2 family protein expression in breast carcinomas in relation to apoptosis. J Pathol, 187: 410-415.

37. Berardo MD, Elledge RM, de Moor C, et al(1998) :-Bcl-2 and apoptosis in lymph node positive breast carcinoma. Cancer, 82: 1296-1302.

38. Honma1N, Horii R, Ito Y, et al (2015):-Differences in clinical importance of Bcl-2 in breast cancer according to hormone receptors status or adjuvant endocrine therapy. BMC Cancer , 15:698 DOI 10.1186/s12885-015-1686-y

39. Dawson S, Makretsov N, Blows FM, et al (2010) :- BCL2 in breast cancer: a favourable prognostic marker across molecular subtypes and independent of adjuvant therapy received .British Journal of Cancer ;103, $668-675$

40. Cory S, Huang DC, Adams JM, et al (2003) :- The Bcl-2 family: roles in cell survival and oncogenesis. Oncogene 22: 8590-8607 
41. Silvestrini R, Benini E, Veneroni S,et al (1996) :-P53 and bcl-2 expression correlates with clinical outcome in a series of node-positive breast cancer patients. J Clin Oncol,14: 1604-1610.

42. Lipponen P, Pietilainen T, Kosma VM, et al(1995):- A poptosis suppressing protein bcl-2 is expressed in welldifferentiated breast carcinomas with favourable prognosis. J Pathol 177: 49-55.

43. Pietenpol JA, Papadopoulos N, Markowitz S, et al (1994) :-Paradoxical inhibition of solid tumor cell growth by bcl2. Cancer Res 54: 3714-3717

44. Wang TT and Phang JM (1995):- Effects of estrogen on apoptotic pathways in human breast cancer cell line MCF-7. Cancer Res 55: 2487-2489

45. Krajewski S, Thor AD, Edgerton SM , et al (1997): Analysis of Bax and Bcl-2 expression in p53-immunopositive breast cancers. Clin Cancer Res ，Feb; 3(2): 199-208.

46. Krajewski S, Blomqvist C, Franssila K, et al (1995): Reduced expression of proapoptotic gene BAX is associated with poor response rates to combination chemotherapy and shorter survival in women with metastatic breast adenocarcinoma. Cancer Res ; 55(19): 4471-78

47. Pluta P and Smolewsk P (2011): Significance of Bax expression in breast cancer patients. Polish Journal of Surgery, $83,10,549-553$. 